H. Saito

Nagoya Math. J.

Vol. 94 (1984), 1-41

\title{
THE HODGE COHOMOLOGY AND CUBIC EQUIVALENCES
}

\author{
HIROSHI SAITO
}

In 1969, Mumford [8] proved that, for a complete non-singular algebraic surface $F$ over the complex number field $C$, the dimension of the Chow group of zero-cycles on $F$ is infinite if the geometric genus of $F$ is positive. To this end, he defined a regular 2 -form $\eta_{f}$ on a non-singular variety $S$ for a regular 2 -form $\eta$ on $F$ and for a morphism $f: S \rightarrow S^{n} F$, where $S^{n} F$ is the $n$-th symmetric product of $F$, and he showed that $\eta_{f}$ vanishes if all 0 -cycles $f(s), s \in S$, are rationally equivalent. Roitman [9] later generalized this to a higher dimensional smooth projective variety $V$. For $\omega \in H^{0}\left(V, \Omega_{V}^{q}\right)$, he has defined $\omega_{n} \in H^{0}\left(S^{n} V, \Omega^{q}\right)$ and proved that $\omega_{n}$ has the following property: if $f, g: S \rightarrow S^{n} V$ are morphisms such that the zero cycles $f(s)$ and $g(s)$ are rationally equivalent for every $s \in S$, then $f^{*} \omega_{n}=g^{*} \omega_{n}$. We may say this property, roughly, like this: $f^{*} \omega_{n}$ cannot distinguish the rational equivalence relation. The rational equivalence is the finest equivalence relation among the adequate equivalence relations (cf. [12]). We can therefore pose the problem: which equivalence relation can $f^{*} \omega_{n}$ distinguish and which one can $f^{*} \omega_{n}$ not?

On the other hand, Samuel has defined the cubic equivalences in [12]. Consider an algebraic family of cycles on a smooth projective variety $V$ over an algebraically closed field $k$, parametrized by a smooth variety $S$. We can regard this family as a "function" on $S$ with values in the set of cycles on $V$. A cycle algebraically equivalent to zero can be considered as the difference of values at two points for an appropriate "function" on a smooth projective curve. We shall assume that the parameter space $S$ is a product of two curves $C_{1} \times C_{2}$. Then we can define a difference of the second order: take two points $a_{i}^{(0)}$ and $a_{i}^{(1)}$ on $C_{i}, i=1,2$, respectively, and form a difference of values at $\left(a_{1}^{(0)}, a_{2}^{(0)}\right)$ and $\left(a_{1}^{(1)}, a_{2}^{(0)}\right)$. We also form a difference between $\left(a_{1}^{(0)}, a_{2}^{(1)}\right)$ and $\left(a_{1}^{(1)}, a_{2}^{(1)}\right)$. The difference of the second order is the difference of these two differences.

Received January 29, 1981.

Revised March 16, 1981. 
Similarly we can consider the differences of higher order. The totality of differences of the $\ell$-th order with values in the cycles on $V$ defines an equivalence relation on the set of cycles on $V$, which we call the b-cubic equivalence relation. This equivalence relation behaves nicely, i.e., if $X$ and $X^{\prime}$ are cycles on $V, b$-cube equivalent, then, for a morphism $f$, the direct images $f_{*} X$ and $f_{*} X^{\prime}$, or the pull-backs $f^{*} X$ and $f^{*} X^{\prime}$, if defined, are also $\ell$-cube equivalent.

Now we assume, for simplicity, $k=C$ and let $f$ be a family of effective $r$-cycles on $V$ parametrized by $S$. To an element $\omega \in H^{r+\ell, r}(V)=$ $H^{r}\left(V, \Omega^{r+\ell}\right)$, we can attach a regular $\ell$-form $f^{\sharp} \omega$ on $S$, generalizing Roitman's $f^{*} \omega_{n}$. Then one of our theorems (5.7) replies partially to the problem raised above, by saying that $f^{\sharp} \omega$ cannot distinguish the $\ell^{\prime}$-cubic equivalence relation for $\ell<\ell^{\prime}$; but $f^{\sharp} \omega$ happens to distinguish the $\ell$-cubic equivalence, cf. (5.14). Our answer is, however, incomplete, as the following example indicates: if $S$ is a point and $\ell=0$, then $f^{\sharp} \omega$ is the dual of the fundamental class of $\operatorname{Im}(f)$, and it occurs that $f^{\sharp} \omega=g^{\sharp} \omega$ but $\operatorname{Im}(f)$ and $\operatorname{Im}(g)$ are not algebraically equivalent, i.e., not 1-cube equivalent by the example of Griffiths [3, 7].

Regarding the Hodge cohomology $H^{* *}(V)$ as a functor from the category of smooth projective varieties to that of $C$-vector spaces, we shall denote by ${ }^{\sharp} H^{* *}(V)$ the minimum subfunctor covariant and contravariant of $H^{* *}(V)$, stable by the multiplication, and containing all the fundamental classes of algebraic cycles and $H^{0,1}(V)$. Then ${ }^{\sharp} H^{p, q}(V)=0$ for $p>q$ and ${ }^{\sharp} H^{p-1, p}(V)$ can be interpreted as the tangent space for the algebraic part of the $p$-th intermediate Jacobian. If $g r^{\ell} \mathrm{CH}^{p}(V)$ is the set of cycles on $V$ of codimension $p, \ell$-cube equivalent to zero modulo $(\ell+1)$-cubic equivalence, then the theorem (5.14) states that ${ }^{\sharp} H^{p-\ell, p}(V)$ $\neq 0$ implies $g r^{\ell} C H^{p}(V) \otimes \boldsymbol{Q} \neq 0$. This shows that a part ${ }^{\sharp} H^{p-\ell, p}(V)$ of the Hodge cohomology $H^{p-\ell, p}(V)$ controls the structure of Chow group of codimension $p$.

In Chapter 1, we describe relations between cycles on $S \times V$ and the rational maps from $S$ to the Chow schemes of $V$, which are reformulations of well-known facts about Chow schemes. In Chapter 2, we generalize a part of Roitman's theory to the case of cycles of intermediate dimension and see that $f^{\sharp} \omega$ never distinguishes the rational equivalence. The statements of the propositions in this Chapter and their proofs, which we refer to [9] with slight modification, are the prototypes of 
theorems in Chapter 5. In Chapter 3, we define cubic equivalences and describe the elementary properties of them. We also remark an interesting theorem essentially due to Swan: if the ground field is the algebraic closure of a finite field, then the theorem of square holds for arbitrary cycles on a smooth projective variety. In Chapter 4, we show that the set of rational maps from a projective scheme into another forms a scheme as $\operatorname{Hom}_{S}(X, Y)$ does, for use in the following chapter. Chapter 5 is devoted mainly to the statements and the proofs of the theorems mentioned above.

\section{$\S 1$.}

(1.1) Let $k$ be an arbitrary field, and $V$ be a geometrically integral algebraic $k$-scheme, $r$ an integer $\geq 0$. By an $r$-cycle on $V$, we understand a formal sum $\sum n_{i} X_{i}$ of $r$-dimensional integral subschemes $X_{i}$ of $V$, where $n_{i} \in Z$. If $n_{i} \geq 0, X$ is said to be effective. We say also $X$ a cycle on $V$ of codimension $(m-r)$ where $m=\operatorname{dim} V$. If $K / k$ is a field extension, we have a morphism $\varphi_{K / k}$ of schemes $V_{K}=V \times{ }_{k} K \rightarrow V$. Given an integral subscheme $X$ of $V$, we define $\varphi_{K / k, V}^{*}(X)$ by

$$
\varphi_{K / k, V}^{*}(X)=\sum \operatorname{length}\left(\mathcal{O}_{X_{K}, y}\right) \bar{y},
$$

where $y$ runs over the maximal points of $X_{K}$. By linearity, we can extend $\varphi_{K / k, V}^{*}$ to a map from the set of cycles on $V$ to those on $V_{K}$. A cycle $X^{\prime}$ on $V_{K}$ is said to be rational over $k$ if there exists a cycle $X$ on $V$ such that $X^{\prime}=\varphi_{K / k, V}^{*}(X)$. Note that such $X$ is unique.

(1.2) Suppose further $V$ projective over $k$ and fix an embedding $V \smile P$ over $k$ and take an algebraically closed field $\Omega \supset k$. Then we can speak of the degree of an $r$-cycle on $V$ and the Chow scheme $C_{r}\left(V_{\Omega}\right)_{d}$ of effective $r$-cycles of degree $d$ on $V_{\Omega} . \quad C_{r}\left(V_{\Omega}\right)_{d}$ is defined over $k$, i.e., there exists a scheme $C_{r}(V)_{d}$ over $k$ such that $C_{r}\left(V_{\Omega}\right)_{d}=C_{r}(V)_{d(\Omega)}$. The $\Omega$-valued points of $C_{r}\left(V_{\Omega}\right)_{d}$ correspond bijectively to the set of $r$-cycles on $V_{\Omega}$ of degree $d$. Let $X$ be an effective $r$-cycle on $V_{\Omega}$ of degree $d$ and $\bar{x}$ the corresponding $\Omega$-valued point of $C_{r}(V)_{d}$. We say that $\bar{x}$ is rational over $k$ if $\kappa(x)=k$ for the image $x$ of $\bar{x}$ by $C_{r}\left(V_{\Omega}\right)_{d}=C_{r}(V)_{(\Omega)} \rightarrow C_{r}(V)_{d}$. If $X$ is rational over $k$, then $\bar{x}$ is rational over $k$; conversely if $\bar{x}$ is rational over $k$, then there exists a purely inseparable finite extension $K$ of $k$ such that $X$ is rational over $K$ (cf. [11], p. 47). Therefore if char $k=0$ and $\bar{x}$ is rational over $k, X$ is rational over $k$. 
(1.3) If $K / k$ is a field extension, and if $T$ is a variety over $k$ such that $k(T)=K$ then for $r$-cycle $X^{\prime}$ on $V_{K}$, we can define an $r$-cycle $\bar{X}^{\prime}$ on $T \times V$ by the following procedure: if $X^{\prime}$ is an integral subscheme of $V_{K}, \bar{X}^{\prime}=$ the schematic image of $X^{\prime}$ by $V_{K} \rightarrow T \times V$. For general $X^{\prime}$, we define $\bar{X}^{\prime}$ by linearity. If $X$ is an integral subscheme of $T \times V$ such that $X \subset T \times V \rightarrow T$ is surjective, then $X_{K}$ is an integral subscheme of $V_{K}$. For $X=\sum n_{i} X_{i}$, if $X_{i} \subset T \times V \rightarrow T$ are surjective for all $i, n_{i} \neq 0$, we say that $X$ is non-degenerate on $T$. For a cycle $X$ on $T \times V$ non-degenerate on $T$, we can define the cycle $X_{K}$ by linearity. Then $X^{\prime} \mapsto \bar{X}^{\prime}$ and $X \mapsto X_{K}$ define a one-to-one correspondence between the set of $r$-cycles on $V_{K}$ and the set of cycles on $T \times V$ of codimension $p=m-r$, non-degenerate on $T$.

For a cycle $X$ on $T \times V$ nondegenerate on $T$, if $d$ is the degree of the cycle $X_{K}$ on $V_{K}, X_{K}$ determines a $K$-rational point of $C_{r}(V)_{d}$, hence a $k$-rational map $f: T \cdots C_{r}(V)_{d}$. Conversely, given a $k$-rational map $f: T \cdots C_{r}(V)_{d}$, we denote by $x$ the image of the generic point of $T$. The corresponding cycle is rational over a purely inseparable extension $K^{\prime}$ of $K$. If $K^{\prime}=K$ (true when char $k=0$ ), let $X^{\prime}$ be the corresponding cycle on $V_{K}$ and $\bar{X}$ the cycle on $T \times V$, the "closure" of $X^{\prime}$; then $\bar{X}$ is (effective and) non-degenerate on $T$ and $\bar{X}_{K}=X^{\prime}$ is of degree $d$. If $f: T$ $\ldots C_{r}(V)_{d}$ is obtained by a cycle on $T \times V$ as above, then we may assume $K^{\prime}=K$ and $\bar{X}=X$. Starting from a rational map $\bar{f}: T \cdots C_{r}(V)_{d}$ and if $K^{\prime}=K$, we obtain a cycle $\bar{X}$ on $T \times V$. The rational map induced from the cycle $\bar{X}$ is the rational map $f$. If char $k=0$, it therefore follows that the correspondences $X \mapsto f$ and $f \mapsto \bar{X}$ define bijective maps between the set of rational maps of $T$ to $C_{r}(V)_{d}$ and the set of cycles $X$ on $T \times V$ of codimension $p$ which is non-degenerate on $T$ and such that $X(t)$ for general $t \in T$ is degree $d$.

Remark (1.3.1). Let $f: T \ldots C_{r}(V)_{d}$ be a rational map and $K^{\prime}$ a purely inseparable extension of $k(T)$ over which the corresponding cycle is rational. If there exists a smooth projective variety $T^{\prime}$ with the function field $K^{\prime}$, we have a cycle $\bar{X}$ on $T^{\prime} \times V$ with the properties described above. Note that if $C$ is a projective curve and if $K^{\prime} / k(C)$ is a purely inseparable finite extension, there exists a smooth projective model $C^{\prime}$ of $K^{\prime}$; and if $C$ is of genus zero, so is $C^{\prime}$. Taking this fact into account, the reader will convince oneself that the propositions (2.2) and (2.3), and the theorems (5.5) and (5.6) below hold even in the positive characteristic 
case (provided that $k$ is uncountable if necessary).

(1.4) Let $k$ be algebraically closed, $V$ smooth projective, $T$ smooth quasi-projective and $X$ a cycle on $T \times V$ of codimension $p$, non-degenerate on $T$. There exists a normal projective variety $\bar{T}$ containing $T$ as an open set. Let $\bar{X}$ be the closure of $X$ in $\bar{T} \times V$, and $d$ the degree of the cycle $\bar{X}_{k(\bar{T})}$ on $V_{k(\bar{T})} . \quad \bar{X}$ induces a $k$-rational map $f^{\prime}: \bar{T} \cdots C_{r}(V)_{d}$, or $f: T$ $\ldots C_{r}(V)_{d}$. If $t \in \bar{T}$ is a closed point such that $\bar{X}(t)$ is defined, then $f^{\prime}(t)$ is defined and corresponds to $\bar{X}(t)$ (cf. [11], p. 107, and Zariski Main Theorem). Conversely, if a closed point $t_{0} \in T$ is in $\operatorname{dom}(f)$, then $\bar{X}\left(t_{0}\right)$ is defined. In fact, let $Y$ be the cycle on $V$ corresponding to $f\left(t_{0}\right)$. Since $T \times V$ is smooth, if $\bar{X}\left(t_{0}\right)$ is not defined, $\operatorname{Supp}(\bar{X}) \cap\left(t_{0} \times V\right)$ is of dimension $>r$. Hence there exists an irreducible component $X_{i}$ of $\operatorname{Supp}(\bar{X})$ whose fibre over $t_{0}$ is not in Supp $Y$. Let $C^{\prime}$ be an irreducible curve in the component such that $C^{\prime}$ has a point $x \in V$ not in Supp $Y$ and $C^{\prime}{ }_{\left(T_{0}\right)} \neq \phi$, where $T_{0}$ is the open subset in $T$ of points $t \in T$ such that $\bar{X}(t)$ is defined. We denote by $C$ the image curve of $C^{\prime}$ on $\bar{T}$ and by $\bar{C}$ its normalization $\varphi: \bar{C} \rightarrow \bar{T}$. Putting $\varphi_{0}: \bar{C}_{0}=\bar{C}_{\left(T_{0}\right)} \rightarrow T_{0}$, we have a cycle $X_{0}=\left(\varphi_{0} \times \mathrm{id}\right)^{*}\left(X_{T_{0} \times V}\right)$ on $C_{0} \times$ $V$ such that if $\bar{X}_{0}$ is its closure in $\bar{C} \times V$, we have $\bar{X}_{0}(s)=\bar{X}\left(\varphi_{0}(s)\right)$ for all $s \in \bar{C}_{0}$. Moreover the rational map induced by the cycle $\bar{X}_{0}$ is the morphism $\bar{C} \stackrel{\varphi}{\rightarrow} \bar{T} \stackrel{f^{\prime}}{\rightarrow} C_{r}(V)_{d}$, hence the point $f^{\prime} \circ \varphi(s) \in C_{r}(V)_{d}$ corresponds to the cycle $\bar{X}_{0}(s)$ for $s \in \bar{C}$. But if $s_{0}$ is a point with $\varphi\left(s_{0}\right)=t_{0}, \operatorname{Supp}\left(\bar{X}_{0}\left(t_{0}\right)\right)$ will contain the point $x$, whence $\bar{X}_{0}\left(s_{0}\right) \neq Y$. This contradicts the fact $f \circ \varphi\left(s_{0}\right)=f\left(t_{0}\right)$.

(1.5) Let $T$ be smooth projective over $k$ and $\psi: T \cdots C_{r}(V)_{d}$ a rational map corresponding to a cycle $X$, and $t \in T$ a point. If $C$ is a smooth projective curve and $f: C \rightarrow T$ is a morphism such that $f(C) \cap \operatorname{dim} \psi \neq \phi$, then we have a rational map $g: C \stackrel{f}{\rightarrow} T \stackrel{\Downarrow}{\rightarrow} C_{r}(V)_{d}$, which in fact is a morphism. If $a \in C$ is a point with $f(a)=t$, then as a cycle on $V, g(a) \in$ $C_{r}(V)_{d}$ is uniquely determined up to rational equivalence, not depending on the choice of $C, f$ and $a$, and is in the rational equivalence class $x(t)$, where $x \in C H^{p}(T \times V)$ is the class of $X$ : If $\gamma$ is a zero-cycle on $T$ supported by dom $\psi$, by $\left[\psi_{*}(\gamma)\right]$ we shall understand the cycle $\sum n_{i}\left[\psi\left(t_{i}\right)\right]$, where $\gamma=\sum n_{i}\left(t_{i}\right) \quad\left(n_{i} \in Z\right)$ and $\left[\psi\left(t_{i}\right)\right]$ is the cycle on $V$ corresponding to the Chow point $\psi\left(t_{i}\right)$. Note first that if $\gamma$ and $\gamma^{\prime}$ are 0 -cycles rationally equivalent on $T$, if $\operatorname{dim} T=1$ (hence $\psi$ is a morphism) and if $\psi$ corresponds to a cycle on $T \times V$, then $\psi_{*}(\gamma)$ and $\psi_{*}\left(\gamma^{\prime}\right)$ are 
rationally equivalent. Now we return to the general case. There exists a cycle $\gamma$ on $C$ supported by $f^{-1}(\operatorname{dom} \psi)$ such that $\gamma$ and the cycle $(a)$ are rationally equivalent. Then $[g(a)]_{\text {rat }}\left[g_{*}(\gamma)\right]=\left[\psi_{*}\left(f_{*}(\gamma)\right)\right]=x\left(f_{*}(\gamma)\right),(t)=$ $f_{*}(a) \overbrace{\text { rat }} f_{*}(\gamma)$ on $T$, hence the class of $[g(a)]=x\left(f_{*}(\gamma)\right)=x(t)$.

\section{$\S 2$.}

(2.1) Let $V$ be a smooth projective variety of dimension $m$ over an algebraically closed field $k$ of characteristic 0 , with an embedding $V \subset \boldsymbol{P}_{N}$, $C_{r}(V)_{d}$ its Chow scheme of effective $r$-cycles of degree $d$ on $V$. We put

$$
E_{\mathrm{rat}}^{p}(V)_{d}=\left\{(X, Y) \in C_{r}(V)_{d} \times C_{r}(V)_{d} ; X_{\text {rat }} Y \text { on } V\right\} .
$$

Proposition (2.2). $\quad E_{\mathrm{rat}}^{p}(V)$ is a countable union of closed subsets of $C_{r}(V)_{d} \times C_{r}(V)_{d}$. More precisely, for integers $\pi \geq 0, d^{\prime}, d^{\prime \prime}>0$, let

$$
\begin{aligned}
& E_{\mathrm{rat}}^{p}(V)_{d}^{\pi, d, d^{\prime \prime}} \\
& \quad=\left\{(X, Y) ; \begin{array}{l}
\exists f \in \operatorname{Hom}^{\pi}\left(\boldsymbol{P}_{1}, C_{r}(V)_{d^{\prime}} \times C_{r}(V)_{d^{\prime \prime}}\right) \text { such that } \\
X+\mathrm{pr}_{1} \circ f(0)+\mathrm{pr}_{2} \circ f(\infty)=Y+\mathrm{pr}_{1} \circ f(\infty)+\mathrm{pr}_{2} \circ f(0)
\end{array}\right\} .
\end{aligned}
$$

Then $E_{\mathrm{rat}}^{p}(V)_{d}$ is the union of $E_{\mathrm{rat}}^{p}(V)_{d}^{\pi, d^{\prime} d^{\prime \prime}}$ and the closures of the latter subsets in $C_{r}(V)_{d} \times C_{r}(V)_{d}$ are contained in $E_{\mathrm{rat}}^{p}(V)_{d}$.

The proof is similar to that of [9], Theorem 1, replacing $S^{n} X$ by $C_{r}(V)_{d}$.

Note that if an integral subvariety $F$ of $\boldsymbol{P}_{N}$ is a countable union of closed subsets $F_{n}$, then there exists an $n$ such that $F=F_{n}$ provided that $k$ is uncountable (This holds even if char $k \neq 0$ ).

Proposition (2.3). If $k$ is uncountable and $f, g: T \rightarrow C_{r}(V)$ are two morphisms from a smooth quasi-projective variety $T$ to the Chow scheme such that for all $t \in T, f(t)$ and $g(t)$ are rationally equivalent, then there exists a smooth quasiprojective variety $S$, a dominant morphism $e: S \rightarrow T$ and a morphism $H: P_{1} \times S \rightarrow C_{r}(V)_{d^{\prime}} \times C_{r}(V)_{d^{\prime \prime}}$ such that

$$
f \circ e+\left(\left.\mathrm{pr}_{1} \circ H\right|_{0 \times S}\right)+\left(\left.\mathrm{pr}_{2} \circ H\right|_{\infty \times S}\right)=g \circ e+\left(\left.\mathrm{pr}_{1} \circ H\right|_{\infty \times S}\right)+\left(\left.\mathrm{pr}_{2} \circ H\right|_{0 \times S}\right) \text {, }
$$

where " $+"$ denotes the summation on the Chow scheme which are morphisms of the form $C_{r}(V)_{d} \times C_{r}(V)_{d^{\prime}} \rightarrow C_{r}(V)_{d+d^{\prime}}$.

Again the proof is similar to [9], Theorem 2.

(2.4) We shall recall the definitions and elementary properties of the 
Hodge cohomology. For a smooth quasi-projective variety $U$, we set

$$
H^{p, q}(U)=H^{q}\left(U, \Omega^{p}\right) .
$$

Then if $f: U \longrightarrow V$ is a morphism of such varieties, we obtain a map

$$
f^{*}: H^{p, q}(V) \longrightarrow H^{p, q}(U)
$$

with the functorial property. If $f$ is proper, i.e., projective, we can define a map*)

$$
f_{*}: H^{p, q}(U) \longrightarrow H^{p-d, q-d}(V)
$$

where $d=\operatorname{dim} U-\operatorname{dim} V$. If $U$ and $V$ are projective, then $f^{*}$ and $f_{*}$ are dual provided that $H^{p, q}(U)$ and $H^{p-d, q-d}(V)$ are regarded as the duals of $H^{n-p, n-q}(U)(n=\operatorname{dim} U)$ and $H^{m-p, m-q}(V)(m=\operatorname{dim} V=n-d)$ respectively by Serre duality. Let $V_{0}$ be an open subscheme of $V$ and consider the Cartesian diagram:

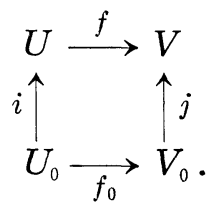

Then the diagram

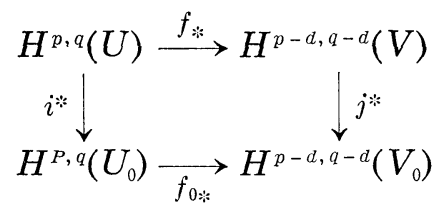

commutes.

The direct sum of $H^{p, q}(U)$ for $0 \leq p, q \leq \operatorname{dim} U$ has a bigraded (anticommutative) ring structure, and $f^{*}$ is a ring homomorphism. For a proper morphism $f: U \rightarrow V$, the projection formula holds, i.e., $f_{*}\left(x . f^{*}(y)\right)=f_{*}(x) . y$ for $x \in H^{* *}(U)$ and $y \in H^{* *}(V)$.

To a closed integral subscheme $Z$ of $U$ of codimension $p$, we can attach an element $\{Z\}$ of $H^{p, p}(U)$, called the fundamental class of $Z$.**) By linearity we can attach an element $\{Z\}$ of $H^{p, p}(U)$ to a cycle $Z$ of

*) cf. [4]. The problem is to define $f_{*}$ for a closed immersion $f$. Use the isomorphism $\mathscr{E}_{x t_{V}^{i}}\left(\mathscr{O}_{U}, \Omega_{V}^{p-d}\right)=\omega \otimes \Omega_{V}^{p-d}(i=-d), 0(i \neq-d)$, where $\omega$ is $\wedge^{-d}$ of the normal bundle of $U$ in $V$ [6].

**) Define, for example, $\{Z\}=(-1)^{p-1} c_{p}\left(O_{z}\right) /(p-1)$ !, where $c_{p}(?)$ is the $p$-th Chern class of ?; note that $\operatorname{ch}\left(\mathrm{O}_{Z}\right)=\{Z\}+$ "higher terms" so that the compatibility with pull-back, or with multiplication is obvious. The compatibility with direct image results from Riemann-Roch theorem, cf. [4], p. 151. 
codimension $p$ on $U$. If $Z$ and $Z^{\prime}$ are cycles on $U$ properly intersecting, then $\left\{Z . Z^{\prime}\right\}=\{Z\}$. $\{Z\}$. If $f: U \rightarrow V$ and $Y$ is a cycle on $V$ such that $f^{*} Y$ is defined, then $\left\{f^{*} Y\right\}=f^{*}\{Y\}$; if $f$ is proper, and $X$ is a cycle on $U$, $\left\{f_{*} X\right\}=f_{*}\{X\}$. Hence, if $Z \underset{\text { alg }}{ } 0$, then $\{Z\}=0$.

Let $V$ be a smooth projective variety and $f: T \rightarrow C_{r}(V)_{d}$ a morphism from a smooth quasi-projective variety into the Chow scheme of $V$, corresponding to a cycle $X$ on $T \times V$ non-degenerate on $T$. For $\omega \in H^{r+\ell, r}(V)$ $(\ell \geq 0)$. we define $f^{\sharp} \omega$ by

$$
f^{\sharp} \omega=\operatorname{pr}_{T^{*}}\left(\{X\} . p_{\nu}^{*} \omega\right)=:\{X\}(\omega)=X(\omega) .
$$

Hence we obtain a map

$$
f^{\sharp}: H^{r+\ell, r}(V) \longrightarrow H^{\ell, 0}(T) .
$$

(2.4.2) If $g: S \rightarrow T$ is a morphism, we have $(f \circ g)^{\sharp}=g^{*} f^{*}$. In fact $\left(g \times \mathrm{id}_{V}\right) * X$ is defined, cf. (1.4), and corresponding to $f \circ g$, and we get

$$
\begin{aligned}
(f \circ g)^{\sharp} \omega & \left.=\operatorname{pr}_{S^{*}}^{\prime}\left\{\left(g \times \mathrm{id}_{V}\right)^{*}(X)\right\} . \operatorname{pr}_{V}^{*} \omega\right) \\
& =\operatorname{pr}_{S^{*}}^{\prime}\left(\left(g \times \mathrm{id}_{V}\right)^{*}\{X\} .\left(g \times \mathrm{id}_{V}\right)^{*} \operatorname{pr}_{V}^{*} \omega\right) \\
& =\operatorname{pr}_{S^{*}}^{\prime}\left(\left(g \times \mathrm{id}_{V}\right)^{*}\left(\{X\} . \mathrm{pr}_{V}^{*} \omega\right)\right) \quad \text { (Künneth formula) } \\
& =g^{*}\left(\mathrm{pr}_{T^{*}}\left(\{X\} . \mathrm{pr}_{V}^{*} \omega\right)\right)=g^{*} f^{\sharp} \omega .
\end{aligned}
$$

(2.4.3) If $f: T \rightarrow C_{r}(V)_{d}$ and $g: T \rightarrow C_{r}(V)_{d}$, are morphisms, we have

$$
f+g: T \stackrel{(f, g)}{\longrightarrow} C_{r}(V)_{d} \times C_{r}(V)_{d^{\prime}} \stackrel{\text { “+” }}{\longrightarrow} C_{r}(V)_{d+d^{\prime}} .
$$

Then $(f+g)^{\sharp}=f^{\sharp}+g^{\sharp}$. In fact if $X$ and $Y$ are corresponding to $f$ and $g$, respectively, then $f+g$ corresponds to the cycle $X+Y$, from which the equality follows immediately.

Proposition (2.5). Under the same hypothesis as in (2.3), we have $f^{\sharp}=g^{\sharp}$.

The proof is similar to [9], Theorem 4 .

(2.6) Let $C H(V)$ be the Chow ring and $C H^{p}(V)$ the codimension $p$ part of $C H(V)$. A map $\kappa$ of a smooth quasi-projective variety $T$ to $C H^{p}(V)$ ( $p+r=m=\operatorname{dim} V$ as before) is said to be regular if there exist a smooth quasi-projective variety $S, z \in C H^{p}(S \times V)$ and a commutative diagram

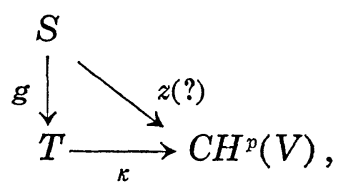


where $\mathrm{g}$ is proper surjective, and $z(s)=i_{s}^{*}(z), i_{s}: V \simeq s \times V \subset S \times V$, the immersion. Cutting $S$ by hyperplanes transversal to the generic fibre of $g$, and choosing an irreducible component dominating $T$ and finally desingularizing it, we may assume that $g$ is generically finite. Let $Z$ be a representative of $z$ and $Z^{\prime}$ the sum (with multiplicity) of components of $Z$ non-degenerate on $S$, and $Z^{\prime \prime}=Z-Z^{\prime}$. Then each component of $Z^{\prime \prime}$ is degenerate on $S$. Since $z^{\prime \prime}(s)=0$ for $s \in S$ where $z^{\prime \prime}$ is the class of $Z^{\prime \prime}$ in $C H(S \times V)$, we may assume that $Z$ is non-degenerate on $S . \quad Z$ is a difference of two effective cycles $Z^{+}$and $Z^{-}$. Let $d_{+}$and $d_{-}$be the degrees of $Z^{+}(s)$ and $Z^{-}(s)$ for general $s \in S$. Then $Z$ defines a rational map $f: S \cdots C_{r}(V)_{a_{+}} \times C_{r}(V)_{d_{-}}$such that for $s \in S$ with $Z(s)$ defined, $Z(s)$ $=\operatorname{pr}_{1} \circ f(s)-\mathrm{pr}_{2} \circ f(s)$. Resolving the indeterminancy of the rational map $f$, we can assume that $f$ is a morphism, and we have a commutative diagram

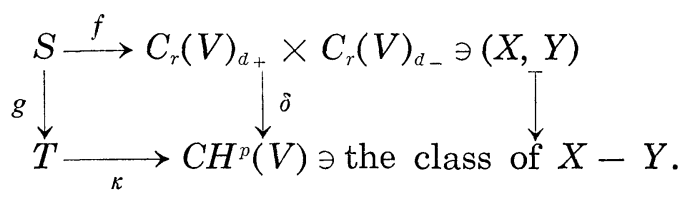

We suppose now that $k$ is uncountable. For $\omega \in H^{r+l, r}(V)$, we put

$$
\kappa^{\sharp} \omega=g_{*}\left(f_{1}^{\sharp} \omega-f_{2}^{\sharp} \omega\right) / \operatorname{deg} g,
$$

where $f_{i}=\operatorname{pr}_{i} \circ f(i=1,2)$. For $\kappa^{\sharp} \omega$ to be well-defined, we must show that the second member is independent of $S, f$ and $g$ chosen. Let

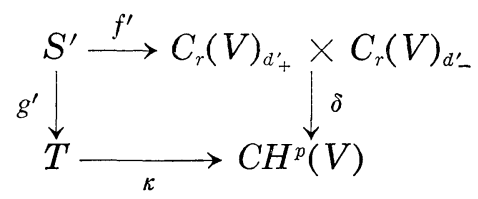

be another one. We can find a smooth quasi-projective variety $S^{\prime \prime}$, morphisms $h: S^{\prime \prime} \rightarrow S$ and $h^{\prime}: S^{\prime \prime} \rightarrow S^{\prime}$ proper surjective and generically finite such that $g \circ h=g^{\prime} \circ h^{\prime}$. So we may assume $S=S^{\prime}$ and $g=g^{\prime}$ because of $h_{*} h^{*}=\operatorname{deg} h$ and $h_{*}^{\prime} h^{*}=\operatorname{deg} h^{\prime}$. Since $g$ is surjective, $f_{1}(s)-f_{2}(s)=$ $f_{1}^{\prime}(s)-f_{2}^{\prime}(s)$ for $s \in S$, i.e., $f_{1}+f_{2}^{\prime}=f_{2}+f_{1}^{\prime}$. The proposition (2.5) and (2.4.3) show that $\kappa^{\sharp} \omega$ is well-defined. We have further $g^{*} \kappa^{\sharp} \omega=f_{1}^{\sharp} \omega-f_{2}^{\sharp} \omega$. In fact, we have $k(T) \subset k(S)$. Let $K$ be a finite Galois extension of $k(T)$ containing $k(S)$, and $S^{\prime}$ be a non-singular model of $K$ such that there exists a proper morphism $S^{\prime} \rightarrow S$ inducing the inclusion $k(S) \subset k\left(S^{\prime}\right)=K$. If $g^{\prime}$ 
denotes the composite $S^{\prime} \rightarrow S \stackrel{g}{\rightarrow} T$, it is sufficient to show that $g^{\prime *} \kappa^{\sharp} \omega=$ $f_{1}^{\prime \#} \omega-f_{2}^{\prime \prime} \omega$, where $f_{\imath}^{\prime}: S^{\prime} \rightarrow S \stackrel{f_{i}}{\rightarrow} C_{r}(V)_{d_{ \pm}}$. We can therefore assume $k(S) / k(T)$ is a finite Galois extension. Let $\sigma$ be a $k(T)$-automorphism of $k(S)$. Then $\sigma$ induces a rational map ${ }^{t} \sigma: S \ldots S$ such that $g \circ{ }^{t} \sigma=g$. Let $S_{0}^{\prime}$ be the domain of definition of ${ }^{t} \sigma$ and we put $T_{0}=T \backslash g\left(S \backslash S_{0}^{\prime}\right)$, an open subset of $T$ and $S_{0}=g^{-1}\left(T_{0}\right)$ :

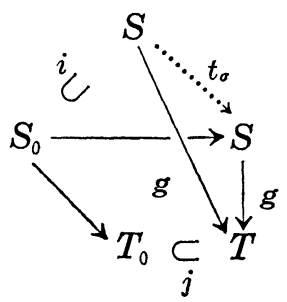

The restriction $g_{0}: S_{0} \rightarrow T_{0}$ of $g$ to $S_{0}$ is proper surjective. Setting $\kappa^{\prime}=\kappa \circ j$ and ${ }^{t} \sigma_{0}: S_{0} \stackrel{i}{\rightarrow} S \stackrel{t_{\sigma}}{\rightarrow} S$, we have two morphisms $f \circ i, f \circ{ }^{t} \sigma_{0}: S_{0} \rightarrow C_{r}(V)_{d_{+}} \times$ $C_{s}(V)_{d_{-}}$covering $\kappa^{\prime}$. The argument above shows

i.e.,

$$
\begin{aligned}
& \left(f_{1} \circ i\right)^{\sharp} \omega-\left(f_{2} \circ i\right)^{\sharp} \omega=\left(f_{1} \circ{ }^{t} \sigma_{0}\right)^{\sharp} \omega-\left(f_{2} \circ{ }^{t} \sigma_{0}\right)^{\sharp} \omega, \\
& i^{*}\left(f_{1}^{\sharp} \omega-f_{2}^{\sharp} \omega\right)={ }^{t} \sigma_{0}^{*}\left(f_{1}^{\sharp} \omega-f_{2}^{\sharp} \omega\right),
\end{aligned}
$$

which in turn implies $f_{1}^{\#} \omega-f_{2}^{\#} \omega$ viewed as a rational $\ell$-form on $S$ is invariant under the action of the Galois group. There is therefore a rational $\ell$-form $\omega^{\prime}$ on $T$ such that $g^{*} \omega^{\prime}=f_{1}^{\#} \omega-f_{2}^{\sharp} \omega$, from which

$$
\omega^{\prime}=g_{*}\left(f_{1}^{\sharp} \omega-f_{2}^{\sharp} \omega\right) / \operatorname{deg} g=\kappa^{\sharp} \omega, \text { i.e., } \quad g^{*} \kappa^{\sharp} \omega=f_{1}^{\sharp} \omega-f_{2}^{\sharp} \omega .
$$

Note that if $Z$ is a cycle on $S \times V$ of codimension $p$ whose components are degenerate on $S$, then $\{Z\}(?): H^{r+\ell, r}(V) \rightarrow H^{\ell, 0}(S)$ is a zero map, since if $S_{0} \subset S$ is an open subset such that $Z_{0}=\left.Z\right|_{S_{0} \times V}$ is zero, then

$$
0=\left\{Z_{0}\right\}(?): H^{r+\ell, r}(V) \longrightarrow H^{\ell, 0}(S) \longrightarrow H^{\ell, 0}\left(S_{0}\right) \text {. }
$$

Thus $\kappa^{\sharp} \omega$ is characterized as follows: if in the diagram (2.6.1), $g$ is generically finite, $\kappa^{\sharp} \omega \in H^{\ell, 0}(T)$ is the element $\omega^{\prime} \in H^{\ell, 0}(T)$ such that $g^{*} \omega^{\prime}=z(\omega)$.

(2.6.2) Suppose $\varphi: T^{\prime} \rightarrow T$ is a morphism. Then $\kappa \circ \psi$ is also regular and

$$
(\kappa \circ \psi)^{\#}=\psi^{*} \circ \kappa^{\sharp} .
$$

(2.6.3) If $U$ is a smooth projective variety and $u \in C H^{r+q}(V \times U)$, we have a map 


$$
u(?): C H^{p}(V) \longrightarrow C H^{q}(U), x \longmapsto u(x)=\operatorname{pr}_{U^{*}}\left(u \cdot \operatorname{pr}_{V}^{*}(x)\right) .
$$

Then $u(?) \circ \kappa: T \rightarrow C H^{q}(U)$ is also regular and

$$
(u(?) \circ \kappa)^{\sharp}=\kappa^{\sharp} \circ{ }^{t} u: H^{n+l-q, n-q}(U) \longmapsto H^{l, 0}(T) .
$$

These assertions follow readily from the above characterization of $\kappa^{\sharp} \omega$.

(2.6.4) If $\kappa_{i}: T \rightarrow C H^{p}(V)(i=1,2)$ are regular maps, then $\kappa_{1}+\kappa_{2}: T$ $\rightarrow C H^{p}(V)$ is also a regular map and $\left(\kappa_{1}+\kappa_{2}\right)^{*}=\kappa_{1}^{\sharp}+\kappa_{2}^{\sharp}$. In fact, let $z_{i} \in$ $C H^{p}\left(S_{i} \times V\right)$ and $g_{i}: S_{i} \rightarrow T$ be proper surjective morphisms such that $\kappa_{i} \circ g_{i}=z_{i}(?)(i=1,2)$. We are immediately reduced to the case $S_{1}=S_{2}$ and $g_{1}=g_{2}$. Then the assertion is evident.

Lemma (2.7). If $\kappa: T \rightarrow C H^{p}(V)$ is regular, then for any $x \in C H^{p}(V)$, $\kappa^{-1}(x)$ in $T$ is a countable union of closed subsets.

In fact we have

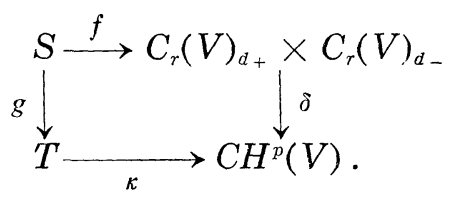

Then $g^{-1}\left(\kappa^{-1}(x)\right)=f^{-1}\left(\delta^{-1}((x))\right.$. If $x$ is the class of a difference of effective cycles $X_{+} \in C_{r}(V)_{d_{+}^{\prime}}$ and $X_{-} \in C_{r}(V)_{d_{-}^{\prime}}$, then

$$
\delta^{-1}(x)=\left\{\left(Y_{+}, Y_{-}\right) \in C_{r}(V)_{d_{+}} \times C_{r}(V)_{d_{-}} ; Y_{+}+X_{-} \underset{\text { ral }}{ } Y_{-}+X_{+}\right\} .
$$

If $d_{+}-d_{-} \neq d_{+}^{\prime}-d_{-}^{\prime}, \kappa^{-1}(x)=\phi$; if $d_{+}-d_{-}=d_{+}^{\prime}-d_{-}^{\prime}$, we have a morphism

$$
\begin{gathered}
F: C_{r}(V)_{t \perp} \times C_{r}(V)_{d_{-}} \longrightarrow C_{r}(V)_{d^{\prime \prime}} \times C_{r}(V)_{d^{\prime \prime}}, \\
\left(Y_{+}, Y_{-}\right) \longmapsto\left(X_{+}+Y_{-}, X_{-}+Y_{+}\right),
\end{gathered}
$$

where $d^{\prime \prime}=d_{+}+d_{-} . \quad \delta^{-1}(x)$ is a countable union of closed subsets in $C_{r}(V)_{d_{+}} \times C_{r}(V)_{d_{-}}$by $\delta^{-1}(x)=F^{-1}\left(E_{\text {rat }}^{p}(V)_{a}\right)$ and by (2.2). This implies that $g^{-1}\left(\kappa^{-1}(x)\right)$ is a countable union of closed subsets of $S$. Since $g$ is proper surjective, $\kappa^{-1}(x)=g\left(g^{-1}\left(\kappa^{-1}(x)\right)\right.$ is a countable union of closed subsets of $T$.

$\S 3$.

(3.1) We shall recall the definition of the cubic equivalences. Let $k$ be an algebraically closed field of arbitrary characteristic, $V$ a smooth projective variety over $k$. 
Definition (3.1.1) cf. [12]. Let $\ell$ be a positive integer. Two cycles $X$ and $Y$ on $V$ of codimension $p$ are said to be $\ell$-cube equivalent and denoted by $X \underset{(\ell)}{\sim} Y$ if there exist $l$ curves (smooth projective over $k$ ) $C_{1}, \cdots C_{\ell}$, a cycle $Z$ on $C_{1} \times \cdots \times C_{\ell} \times V$ of codimension $p$, points $a_{i}^{(0)}$, $a_{i}^{(1)}$ of $C_{i}(i=1, \cdots, \ell)$ such that

$$
\begin{gathered}
Z\left(a_{1}^{\left(i_{1}\right)}, \cdots, a_{\ell}^{\left(\imath_{\ell}\right)}\right) \text { are defined for all } i_{1}, \cdots, i_{\ell}=0,1, \\
X-Y=\sum_{i_{1}, \cdots, i_{\ell}=0,1}(-1)^{i_{1}+\cdots+i_{\ell}} Z\left(a_{1}^{\left(i_{1}\right)}, \cdots, a_{\ell}^{\left(i_{\ell}\right)}\right) .
\end{gathered}
$$

For $\ell=1$, $\ell$-cubic equivalence is no other than the algebraic equivalence. 2-cubic equivalence is also called square equivalence and 3cubic equivalence is called simply cubic equivalence. For convention we say that any two cycles on $V$ are 0 -cube equivalent.

Remark (3.1.4). Our definition is a priori different from Samuel's one because in our definition the objects are defined over $k$, while in Samuel's, they are considered over the universal domain.

Proposition (3.2). 1) The e-cubic equivalence is an adequate equivalence relation (cf. [12]) so that the set of cycles on $V$ modulo b-cubic equivalence is naturally equipped with a structure of commutative rings and has functorial properties; in particular the $\ell$-cubic equivalence is coarser than the rational equivalence.

2) If $\ell^{\prime}<\ell$, the $\ell$-cubic equivalence is finer than the $\ell$-cubic equivalence.

3) If $X$ is a cycle on $V$, $\ell$-cube equivalent to zero and if $X^{\prime}$ is a cycle on $V^{\prime}$, $\ell^{\prime}$-cube equivalent to zero, then the cycle $X \times X^{\prime}$ on $V \times V^{\prime}$ is $\left(\ell+\ell^{\prime}\right)$-cube equivalent to zero.

The proofs, more or less formal, can be found in [12].

(3.3) We denote by $F^{\ell} C H^{p}(V)$ the cycles on $V$ of codimension $p$, $\ell$ cube equivalent to zero modulo rational equivalence and by $F^{\ell} C H^{p}(V)$ the direct sum of $F^{\ell} C H^{p}(V)$ for $0 \leq p \leq \operatorname{dim} V$. We also write $F^{\ell} C H^{p}(V)$ $=F^{\ell} C_{r}(V)$ if $p+r=\operatorname{dim} V$. By definition, $F^{0} C H(V)=C H(V)$. We have thus a descending filtration

$$
C H(V)=F^{0} C H(V) \supset F^{1} C H(V) \supset \cdots \supset F^{\ell} C H(V) \supset F^{\ell+1} C H(V) \supset \cdots .
$$

We put: $\quad g r^{\ell} C H^{p}(V)=g r^{\ell} C H_{r}(V)=F^{\ell} C H^{p}(V) / F^{\ell+1} C H^{p}(V)$.

$$
g r^{*} C H^{*}(V)=\underset{\ell, 0 \leq p \leq \operatorname{dim} V}{\oplus} g r^{\ell} C H^{p}(V) \text {. }
$$


By (3.2.3)), $g r^{*} C H^{*}(V)$ has a structure of bigraded commutative rings, the multiplication being induced by that of $C H(V)$. For a morphism $f: V \rightarrow$ $V^{\prime}, f^{*}: C H\left(V^{\prime}\right) \rightarrow C H(V)$ induces a homomorphism of bigraded rings

$$
f^{*}: g r^{*} C H^{*}\left(V^{\prime}\right) \longrightarrow g r^{*} C H^{*}(V) ;
$$

$f_{*}: C H(V) \rightarrow C H\left(V^{\prime}\right)$ induces a group homomorphism

$$
f_{*}: g r^{*} C H^{*}(V) \longrightarrow g r^{*} C H^{*}\left(V^{\prime}\right)
$$

such that $f_{*}\left(g r^{\ell} C H_{r}(V)\right) \subset g r^{\ell} C H_{r}\left(V^{\prime}\right)$.

Examples (3.4) 1) $g r^{0} \mathrm{CH}^{p}(V)$ is the so-called Néron-Severi group of codimension $p$.

2) $\mathrm{gr}^{1} \mathrm{CH}^{1}(V)$ is isomorphic to the Picard variety of $V$ as groups.

3) We have $g r^{1} \mathrm{CH}_{0}\left(V \times V^{\prime}\right) \simeq g r^{1} \mathrm{CH}_{0}(V) \times g r^{1} C H_{0}\left(V^{\prime}\right)$ which results from the formula

$$
\sum n_{i}\left(\left(x_{i}, y_{i}\right)\right) \underset{(2)}{\widetilde{1}}\left\{\sum n_{i}\left(x_{i}\right)\right\} \times\left(y_{0}\right)+\left(x_{0}\right) \times\left\{\sum n_{i}\left(y_{i}\right)\right\},
$$

where $x_{i}, x_{0} \in V$, and $y_{i}, y_{0} \in V^{\prime}$ and $n_{i}$ are integers such that $\sum n_{i}=0$. In particular, if $V$ is a product of curves,

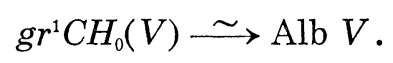

Lemma (3.5) (cf. $[12,13])$. Let $X$ be a cycle on $V$ of codimension $p$ and $\ell$ a positive integer. Then the following conditions on $X$ are equivalent:

a) $X$ is $\ell$-cube equivalent to zero.

b) There exist $\ell$ smooth projective varieties $T_{1}, \cdots, T_{\ell}$ over $k$, a cycle $Z$ on $T_{1} \times \cdots \times T_{\ell} \times V$ of codimension $p$ and points $a_{i}^{(0)}$ and $a_{i}^{(1)}$ on $T_{i}$ $(1 \leq i \leq \ell)$ satisfying the conditions (3.1.2) and (3.1.3) with $Y=0$.

c) The same condition as b), but we further require $T_{i}^{\prime} \mathrm{s}$ to be jacobian varieties.

Clearly c) implies b). a) follows from b) because any two points on a variety can be joined by a smooth projective curve not necessarily in the variety. It therefore suffices to show that a) implies c). Let $C_{i}, Z$ and $a_{j}^{(i)}$ be as in (3.1.1) with $Y=0$. We shall show that there exists a cycle $X^{\prime}$ on $V$ satisfying the condition c) such that $X$ and $X^{\prime}$ are rationally equivalent. We assume that the genera of $C_{i}^{\prime}$ s are positive, since if one of $C_{i}$ is rational, we can take $X^{\prime}=0$. First we suppose $Z$ is a prime cycle, i.e., $Z$ is a variety. Let $J_{i}$ be the jacobian of $C_{i}$ and $g_{i}$ the genus of $C_{i}$. For $1 \leq j_{i} \leq g_{i}$, let 


$$
\psi_{j_{1} \cdots j_{\ell}}: C_{1}^{g_{1}} \times \cdots \times C_{\ell}^{g_{\ell}} \longrightarrow C_{1} \times \cdots \times C_{\ell}
$$

be the product of $j_{i}$-th projections $C_{i}^{g_{i}} \rightarrow C_{i}$ for $i=1, \cdots, \ell$, and $Y$ the reduced subscheme of $C_{1}^{g_{1}} \times \cdots \times C_{\ell}^{g_{\ell}} \times V$ with underlying closed subset

$$
\bigcup_{j_{1}, \cdots, j_{\ell}}\left(\psi_{j_{1}, \cdots, j_{\ell}} \times \mathrm{id}_{V}\right)^{-1}(Z) \text {. }
$$

If $S_{g_{1}}$ is the symmetric group of degree $g_{i}, G=S_{g_{1}} \times \cdots \times S_{g_{\ell}}$ acts on $C_{1}^{g_{1}} \times \cdots \times C_{\ell}^{g_{\ell}} \times V$ and $Y$ is stable by the action of $G$. Moreover

$$
\left(C_{1}^{g_{1}} \times \cdots \times C_{\ell}^{g_{\ell}} \times V\right) / G=S^{g_{1}} C_{1} \times \cdots \times S^{g_{\ell}} C_{\ell} \times V .
$$

Lemma (3.5.1). Let $L / K$ be a finite Galois extension with the Galois group $G$. If $X^{\prime}$ is an algebraic $K$-scheme and $Y$ is a G-stable reduced subscheme of $X=X^{\prime} \underset{K}{\times} L$, then $Y / G$ is a closed reduced subscheme of $X$ and $(Y / G) \underset{K}{\times} L=Y$.

Consider the diagram

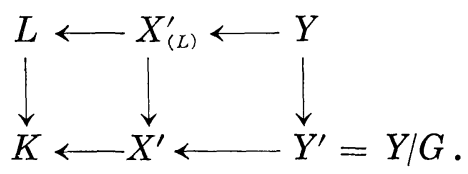

We have the natural morphism $k: Y \rightarrow Y_{(L)}^{\prime}$. We shall show that $k: Y \rightarrow$ $Y_{(L)}^{\prime}$ is an isomorphism, which implies $Y^{\prime}=Y / G \rightarrow X^{\prime}$ is a closed immersion. $Y^{\prime}$ is reduced because of reducedness of $Y . Y_{(L)}^{\prime}$ is also reduced since $L / K$ is a finite separable extension. $\quad Y \stackrel{k}{\rightarrow} Y_{(L)}^{\prime} \rightarrow X$ is a closed immersion by hypothesis. Hence $k: Y \rightarrow Y_{(L)}^{\prime}$ is also a closed immersion, so that it is sufficient to show that $k: Y \rightarrow Y_{(L)}^{\prime}$ is bijective, or surjective. Let $p$ be a point of $Y_{(L)}^{\prime}$. There is a point $q$ of $Y$ such that the image of $q$ in $Y^{\prime}$ is equal to that of $p$, by the surjectivity of $Y \rightarrow Y^{\prime}$. G acts on $Y_{(L)}^{\prime}$ and the actions of $G$ on $Y_{(L)}^{\prime}$ and on $Y$ are compatible with the morphism $k$. Since the action of $G$ on a fibre of $Y_{(L)}^{\prime} \rightarrow Y^{\prime}$ is transitive, there exists a $\sigma \in G$ such that $\sigma \cdot q=p$. As $Y$ is $G$-stable, $p$ is a point of $Y$.

We shall continue the proof of (3.5). Let $\eta$ be the generic point of $C_{1}^{g_{1}} \times \cdots \times C_{\ell}^{g_{\ell}}$. Then $Y_{\eta}$ is a reduced closed subscheme of $V_{\kappa(\eta)}$. Hence if $K=\kappa(\eta)^{G}$, there exists a closed subscheme $Y^{\prime}$ of $V_{K}$ such that $Y^{\prime} \underset{K}{\times} \kappa(\eta)=$ $Y_{\eta}$. If $\eta^{\prime}$ is the generic point of $J_{1} \times \cdots \times J_{\ell}$, then $\kappa\left(\eta^{\prime}\right)=K$; therefore $\bar{Y}_{\eta^{\prime}}^{\prime}=Y^{\prime}$ for the schematic closure $\bar{Y}^{\prime}$ of $Y^{\prime}$ in $J_{1} \times \cdots \times J_{\ell} \times V$. Let $\pi_{i}: C_{i}^{g_{i}} \rightarrow J_{i}$ be the canonical morphism and 


$$
\pi=\pi_{1} \times \cdots \times \pi_{\ell}: C_{1}^{g_{1}} \times \cdots \times C_{\ell}^{g_{\ell}} \longrightarrow J_{1} \times \cdots \times J_{\ell} .
$$

We have $\left\{\left(\pi \times \mathrm{id}_{V}\right)^{-1}\left(\bar{Y}^{\prime}\right)\right\}_{\eta}=Y_{\eta}$. Let $F$ be the union of irreducible components of $\left(\pi \times \mathrm{id}_{V}\right)^{-1}\left(\bar{Y}^{\prime}\right)$ degenerate on $C_{1}^{g_{1}} \times \cdots \times C_{\ell}^{g_{\ell}} ; F$ is a closed subset of $C_{1}^{g_{1}} \times \cdots \times C_{\ell}^{g_{\ell}} \times V$ disjoint from $V_{\kappa\left(\eta^{\prime}\right)}$. If we denote by $U$ the open subset

$$
J_{1} \times \cdots \times J_{\ell} \backslash\left(\operatorname{Im}\left(F \longrightarrow C_{1}^{g_{1}} \times \cdots \times C_{\ell}^{g_{\ell}} \longrightarrow J_{1} \times \cdots \times J_{\ell}\right)\right),
$$

we have

$$
\left(\pi_{U} \times \mathrm{id}_{V}\right)^{-1}\left(\left(\bar{Y}^{\prime} \cap U\right) \times V\right)=\left(Y \cap \pi^{-1}(U)\right) \times V .
$$

Shrinking $U$ if necessary, we can assume that $\pi^{-1}(U) \times V \rightarrow U \times V$ is flat, hence denoting the associated cycles also by the same symbols, we have

$$
\left(\pi_{U} \times \mathrm{id}_{V}\right)^{*}\left(\left(\bar{Y}^{\prime} \mid U\right) \times V\right)=\left(Y \mid \pi^{-1}(U)\right) \times V .
$$

By definition we have $Y=\sum_{j_{1}, \ldots, j_{\ell}}\left(\psi_{j_{1}, \ldots, j_{\ell}} \times \mathrm{id}_{V}\right) *(Z)$, hence for $x \in \pi^{-1}(U)$,

$$
\bar{Y}^{\prime}(\pi(x))=\sum_{j_{1}, \ldots, j_{\ell}} Z\left(\psi_{j_{1}, \ldots, j_{\ell}}(x)\right) .
$$

By virtue of the moving lemma, for any $x \in C_{1}^{g_{1}} \times \cdots \times C_{\ell}^{g_{\ell}}$, we have

$$
\bar{y}^{\prime}(\pi(x))=\sum_{j_{1}, \ldots, j_{\ell}} z\left(\psi_{j_{1}, \ldots, j_{\ell}}(x)\right)
$$

where $\bar{y}^{\prime}$ and $z$ are the ratinoal equivalence classes of $\bar{Y}^{\prime}$ and $Z$, respectively. By linearity, we may suppose that this equality holds for an arbitrary cycle $Z$ on $C_{1} \times \cdots \times C_{\ell} \times V$. Denoting $\bar{a}_{h}^{(i)}=\left(a_{h}^{(i)}, a_{h}^{(0)}, \cdots, a_{h}^{(0)}\right)$ $\in C_{h}^{g_{h}}(1 \leq h \leq \ell, i=0,1)$, we have

$$
\begin{aligned}
\sum_{i_{1}, \cdots, i_{\ell}=0,1} & (-1)^{i_{1}+\cdots+i_{\ell}} \bar{y}^{\prime}\left(\pi\left(\bar{a}_{1}^{\left(i_{1}\right)}, \cdots, \bar{a}_{\ell}^{\left(i_{\ell}\right)}\right)\right) \\
& =\sum_{i_{1}, \cdots, i_{\ell}=0,1}(-1)^{i_{1}+\cdots+i_{\ell}} \sum_{j_{1}, \cdots, j_{\ell}} z\left(\psi_{j_{1}, \cdots, j_{\ell}}\left(\bar{a}_{1}^{\left(i_{1}\right)}, \cdots, \bar{a}_{\ell}^{\left(i_{\ell}\right)}\right)\right) \\
& =\sum_{j_{1}, \ldots, j_{\ell} i_{1}, \cdots, i_{\ell}=0,1}(-1)^{i_{1}+\cdots+i_{\ell}} z\left(\psi_{j_{1}, \cdots, j_{\ell}}\left(\bar{a}_{1}^{\left(i_{1}\right)}, \cdots, \bar{a}_{\ell}^{\left(i_{\ell}\right\rangle}\right)\right) \\
& =\sum_{i_{1}, \ldots, i_{\ell}=0,1}(-1)^{i_{1}+\cdots+i_{\ell}} z\left(a_{1}^{\left(i_{1}\right)}, \cdots, a_{\ell}^{\left(i_{\ell}\right)}\right)=\text { the class of } X
\end{aligned}
$$

since if one of $j_{1}, \cdots, j_{\ell}$ is greater than 1 , say $j_{h}>1$, then

$$
\psi_{j_{1}, \cdots, j_{\ell}}\left(\bar{a}_{1}^{\left(i_{1}\right)}, \cdots, \bar{a}_{\ell}^{\left(i_{\ell}\right)}\right)=\left(\cdots, a_{h}^{(0)}, \cdots\right) .
$$

From the moving lemma we deduce that there exists a cycle $Z^{\prime}$ on $J_{1} \times \cdots \times J_{\ell} \times V$, rationally equivalent $\bar{Y}^{\prime}$ such that $Z^{\prime}\left(\pi\left(\bar{a}_{1}^{\left(i_{1}\right)}, \cdots, \bar{a}_{\ell}^{\left(i_{\ell}\right)}\right)\right)$ are all defined. Putting $\pi_{i}\left(\bar{\alpha}_{i}^{(j)}\right)=a_{i}^{\prime(j)} \in J_{\imath}(j=0,1)$, and 


$$
X^{\prime}=\sum_{i_{1}, \ldots, i_{\ell}=0,1} Z^{\prime}\left(a_{1}^{\prime\left(i_{1}\right)}, \cdots, a_{\ell}^{\prime\left(i_{\ell}\right)}\right),
$$

we have our assertion. If one of $C_{i}$ is rational, let $J_{1}, \cdots, J_{\ell}$ be arbitrary jacobians, $a_{i}^{\prime(0)}$ and $a_{i}^{\prime(1)}$ arbitrary points on $J_{i}$, and $Z^{\prime}=0$. Since $X$ and $X^{\prime}$ are rationally equivalent, there is a cycle $Z^{*}$ on $P_{1} \times V$ non-degenerate on $\boldsymbol{P}_{1}$ (hence the components are flat over $\boldsymbol{P}_{1}$ ) such that $Z^{*}(0)=X$ and $Z^{*}(1)=X^{\prime}$. Consider a rational function on $J_{1} \times \cdots \times J_{\ell}$ defined in a neighbourhood of the finite $\operatorname{set}\left\{\left(a_{1}^{\prime\left(i_{1}\right)}, \cdots, a_{\ell}^{\prime\left(i_{\ell}\right)}\right) ; i_{1}, \cdots, i_{\ell}=0,1\right\}$, with its value 0 at $\left(a_{1}^{\prime(0)}, \cdots, a_{\ell}^{\prime(0)}\right)$, and with its value 1 at the other points of the finite set. Let $\Gamma$ be the closure of its graph in $J_{1} \times \cdots \times J_{\ell} \times \boldsymbol{P}_{1}$ and $Z^{\prime \prime}=\Gamma \circ Z^{*}$, a cycle on $J_{1} \times \cdots \times J_{\ell} \times V$; since $Z^{*}$ is "flat" over $\boldsymbol{P}_{1}$, the cycle is defined. The following first member is defined and

$$
Z^{\prime \prime}\left(a_{1}^{\prime\left(i_{1}\right)}, \cdots, a_{\ell}^{\prime\left(i_{\ell}\right)}\right)=\left\{\begin{array}{l}
Z^{*}(0)=X \text { if } i_{1}=\cdots=i_{\ell}=0 \\
Z^{*}(1)=X^{\prime}, \text { otherwise, }
\end{array}\right.
$$

hence,

$$
\sum_{i_{1}, \ldots, i_{\ell}=0,1}(-1)^{i_{i}+\cdots+i_{\ell}}\left(Z^{\prime}+Z^{\prime \prime}\right)\left(a_{1}^{\prime\left(i_{1}\right)}, \cdots, a_{\ell}^{\prime\left(i_{\ell}\right)}\right)=X^{\prime}+X-X^{\prime}=X,
$$

q.e.d.

(3.6) Let $A$ ke an arbitrary abelian variety over $k$. We set

$$
I_{A}=F^{1} C H_{0}(A),
$$

and let $*$ denote the Pontrjagin product.

LEMma (3.6.1). The elements of $F^{\ell} \mathrm{CH}^{p}(V)$ are the elements of the form $z(\gamma)$, where $z \in C H^{p}(A \times V), \gamma \in I_{A}^{* \ell}$ and $A$ is an abelian variety.

In fact since $I_{A}^{* \ell} \subset F^{\ell} C H_{0}(A), z(\gamma) \in F^{\ell} C H^{p}(V)$. Conversely, let $x \in$ $F^{\ell} C H^{p}(V)$ and $X$ a cycle on $V$ representing $x$. Then $X$ is $\ell$-cube equivalent to zero and by (3.5),

$$
X=\sum(-1)^{i_{1}+\cdots+i_{\ell}} Z\left(a_{1}^{\left(i_{1}\right)}, \cdots, a_{\ell}^{\left(i_{\ell}\right)}\right),
$$

where $Z$ is a cycle on $J_{1} \times \cdots \times J_{\ell} \times V$, and $J_{i}$ are jacobian varieties. Let $A=J_{1} \times \cdots \times J_{\ell}$ and $z$ the class of $Z$. Then

$$
\begin{aligned}
x & =\sum(-1)^{i_{1}+\cdots+i_{\ell}} z\left(a_{1}^{\left(i_{1}\right)}, \cdots, a_{\ell}^{\left(i_{\ell}\right)}\right) \\
& =z\left(\left\{\left(a_{1}^{(0)}\right)-\left(a_{1}^{(1)}\right)\right\} \times \cdots \times\left\{\left(a_{\ell}^{(0)}\right)-\left(a_{\ell}^{(1)}\right)\right\}\right) .
\end{aligned}
$$

But we have 


$$
\begin{aligned}
\left\{\left(a_{1}^{(0)}\right)-\left(a_{1}^{(1)}\right)\right\} & \times \cdots \times\left\{\left(a_{\ell}^{(0)}\right)-\left(a_{\ell}^{(1)}\right)\right\} \\
=\left\{\left(a_{1}^{(0)}, 0, \cdots, 0\right)-\left(a_{1}^{(1)}, 0, \cdots, 0\right)\right\} & \\
& * \cdots *\left\{\left(0, \cdots, 0, a_{\ell}^{(0)}\right)-\left(0, \cdots, 0, a_{\ell}^{(1)}\right)\right\},
\end{aligned}
$$

which belongs to $I_{A}^{* \ell}$, so that $x$ is of the form above.

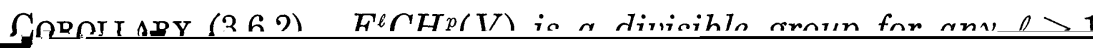


1). i) $\Rightarrow$ ii) follows from (1.4) and the fact that, in the definition (3.1.1), we may suppose $Z$ non-degenerate on $C_{1} \times \cdots \times C_{\ell}$. Assume ii). Replacing $C_{i}^{\prime}$ s by their purely inseparable coverings and lifting the points $a_{j}^{(i)}$ to points on the covering if necessary, we may suppose that $\varphi$ corresponds to a cycle on $C_{1} \times \cdots \times C_{\ell} \times V$; then i) results from (1.4). The equivalence of $\mathrm{i}$ ) and iii) follows similarly, noticing (3.5).

2). Considering the normalization $\bar{C}_{i}$ of $C_{i}$, replacing it by a purely inseparable covering (if necessary) and taking account of the fact that $\Gamma$ lifts to a curve $\bar{T}$ such that the following diagram commutes:

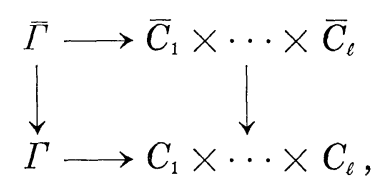

we may assume that $C_{\imath}^{\prime} \mathrm{s}$ are smooth projective and the rational map $\varphi$ corresponds to a cycle on $C_{1} \times \cdots \times C_{\ell} \times V$. Then 2) is a consequence of (1.5) and the fact that the $\ell$-cubic equivalence is coarser than the rational equivalence.

Remark (3.9) Consider the condition on $F^{1} C H^{p}(V)$ :

(B) There exist an abelian variety $A$ and a group isomorphism $h: F^{1} C H^{p}(V) \widetilde{\rightarrow}$ such that for any $u \in C H^{p}(T \times V)$, where $T$ is a smooth projective variety, the map

$$
T \stackrel{u^{\prime}}{\longrightarrow} F^{1} C^{p}(V) \stackrel{\sim}{\longrightarrow} A
$$

is a morphism; here $u^{\prime}$ is a map of the form $t \mapsto u\left((t)-\left(t_{0}\right)\right), t_{0} \in T$.

Then it is immediate that if $F^{2} C H^{p}(V) \neq 0$, then $F^{1} C H^{p}(V)$ never satisfies the condition (B). Note that the condition (B) is a kind of boundedness condition on $C H^{p}(V)$.

\section{$\S 4$.}

(4.1) Suppose that $k$ is an arbitrary field, that $X$ and $Y$ are algebraic $k$-schemes and that $X$ is reduced. Let $f: X \cdots Y$ be a $k$-rational map, $V$ the domain of definition of $f$, an open subset of $X$, and $f_{0}: V \rightarrow Y$ a representative of $f$. Consider the graph $\Gamma \subset V \times \underset{k}{\times}=V \times Y$ of $f_{0}$. Let $U$ be the schematic closure of $\Gamma$ in $X \times Y$. Since $V \simeq \Gamma$ is reduced, (1) $U$ is also reduced. We have a morphism $U \subset X \times Y \rightarrow X$. By base change $V \rightarrow X$, (2) $\Gamma=U_{(V)} \rightarrow V$ is an isomorphism and (3) $U_{(V)}=\Gamma$ is 
dense in $U$. By the definition of rational map, (4) $V$ is dense in $X$. Conversely, suppose given a closed subscheme $U$ of $X \times Y$ and an open subset $V$ of $X$ with properties (1) (4). Then by (2), we have a morphism $f_{0}: V \rightarrow U_{(V)} \rightarrow U \subset X \times Y \rightarrow Y$. The property (4) implies that $f_{0}$ defines a $k$-rational map $f: X \ldots Y$. It is readily checked that the correspondence $\Gamma \rightarrow U$ is one-to-one. We can therefore identify the set of $k$-rational maps from $X$ to $Y$ with the set of closed subschemes $U$ of $X \times Y$ such that there exists an open subset $V$ of $X$ satisfying the properties (1) (4). If a $k$-morphism from $Y$ to $X$ is given, the set of $k$-rational sections is identified with a set of closed subschemes of $Y$ with some similar properties, cf. $(4.2 .1) \sim 4)$ ) below.

(4.2) Let $S$ be a locally noetherian scheme, $X$ and $Y S$-schernes of finite type, and $p: X \rightarrow Y$ an $S$-morphism:

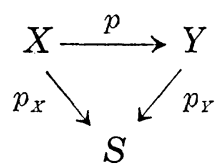

Let $\Gamma_{S \text {-rat }}(X / Y)$ be the set of closed subschemes $U$ of $X$ flat over $S$ such that there exists an open subscheme $V$ of $Y$ satisfying the following conditions:

(4.2.1) $p_{V}^{\prime}: U_{(v)} \rightarrow V$ induced by $p^{\prime}: U \subset X \rightarrow Y$ via base change $V \rightarrow Y$ is an isomorphism;

(4.2.2) For each $s \in S, V_{s} \subset Y_{s}$ is dense,

(4.2.3) $U_{s} \subset X_{s}$ is geometrically reduced for all $s \in S$;

(4.2.4) $\left(U_{(V)}\right)_{s} \subset U_{s}$ is dense for all $s \in S$.

Note that given $U$, there exists the maximum open subscheme $V$ of $Y$ satisfying the above conditions. Given $T \rightarrow S, U \mapsto U_{(T)}$ induces a map

$$
\Gamma_{S-\text { rat }}(X / Y) \longrightarrow \Gamma_{T-\text { rat }}\left(X_{T} / Y_{T}\right) \text {. }
$$

We get therefore a contravariant functor

(4.2.5) $\Gamma_{S \text {-rat }}(X / Y)$ : (loc. noetherian schemes $\left./ S\right) \rightarrow$ (Sets).

Proposition (4.3). Suppose $X$ projective over $S$, and $Y$ projective and flat over $S$. Then the functor $\Gamma_{S \text {-rat }}(X / Y)$ is represented by an open subscheme of the Hilbert scheme $\mathrm{Hilb}_{X / S}$.

It is sufficient to show the following: Suppose $U$ a closed subscheme of $X$ flat over $S$. For some $s \in S$, if there exists an open dense subset 
$V^{\prime}$ of $Y_{s}$ such that $U_{V^{\prime}} \rightarrow V^{\prime}$ is an isomorphism, that $U_{V^{\prime}}$ is dense in $U_{s}$ and that $U_{s}$ is geometrically reduced, then there exist an open subset $S_{0}$ of $S$ containing $s$ such that there exists an open subset $V$ of $Y$ satisfying the conditions $(4.2 .1) \sim 4)$ ) above when we replace $S$ by $S_{0}$. First of all, by [5], IV, 12.1.1, vii, and by the properness of $U$ over $S$, the set of $s \in S$ satisfying the condition (4.2.3) is open. The set $U^{\prime}$ of $x \in U$ such that $x$ is isolated in $p^{\prime-1}\left(p^{\prime}(x)\right)$ is open. Since $p^{\prime}$ is proper and $p^{\prime}\left(U \backslash U^{\prime}\right) \cap V^{\prime}$ $=\phi, Y_{0}=Y \backslash p^{\prime}\left(U \backslash U^{\prime}\right)$ is open in $Y$ and contains $V^{\prime}$. Then $U_{0}=p^{\prime-1}\left(Y_{0}\right)$ is proper over $Y_{0}$ and flat over $S$. The morphism $p_{\left(Y_{0}\right)}^{\prime}: U_{0} \rightarrow Y_{0}$ is finite. Let $U_{0}=\operatorname{Spec} A$ with $A$ a coherent $\mathcal{O}_{Y_{0}}$-Algebra. Define the coherent $\mathcal{O}_{Y_{0}}$-Modules $N$ and $C$ by the exact sequence

$$
0 \longrightarrow N \longrightarrow O_{Y_{0}} \longrightarrow A \longrightarrow C \longrightarrow 0 .
$$

For $y \in V^{\prime}$, the sequence

$$
\mathcal{O}_{Y_{0}, y} / m_{y} \longrightarrow A_{y} / m_{y} A_{y} \longrightarrow C_{y} / m_{y} C_{y} \longrightarrow 0
$$

is exact. $U_{y} \simeq y$ implies $C_{y} / m_{y} C_{y}=0$, hence $C_{y}=0$, i.e., Supp $C \cap V^{\prime}$ $=\phi$. Denote by $Y_{1}$ the open subset $Y_{0} \mid$ Supp $C$ of $Y_{0}$ containing $V^{\prime}$, and set $U_{1}=p^{\prime-1}\left(Y_{1}\right)$. We have a diagam

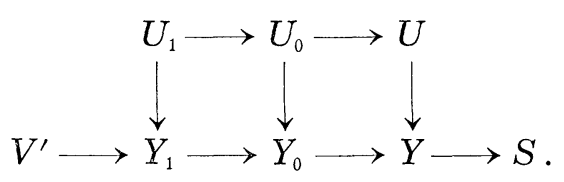

Since $V^{\prime}$ is open in $Y_{s}$, there is an open subset $Y_{0}^{\prime}$ of $Y$ such that $V^{\prime}=$ $Y_{0}^{\prime} \cap Y_{s}$. Replacing $Y_{1}$ by $Y_{1} \cap Y_{0}^{\prime}$, we may assume $V^{\prime}=\left(Y_{1}\right)_{s}$. We denote the restriction of $A$ to $Y_{1}$ also by $A$. Since $U$ is flat over $S, A$ is flat over $S$. We therefore get the exact sequence

$$
0 \longrightarrow N / m_{s} N \longrightarrow \mathcal{O}_{Y_{1}} / m_{s} \mathcal{O}_{Y_{1}} \longrightarrow A / m_{s} A \longrightarrow 0 \text {. }
$$

$\left(U_{1}\right)_{s} \simeq\left(Y_{1}\right)_{s}=V^{\prime}$ implies $N / m_{s} N=0$, or $N=m_{s} N$, a fortiori, $N_{y}=m_{y} N_{y}$ for $y \in V^{\prime}$. Hence $N_{y}=0$ for $y \in V^{\prime}$ or $\operatorname{Supp} N \cap V^{\prime}=\dot{\phi} . \quad V=Y_{1} \mid \operatorname{Supp} N$ is open in $Y$ and contains $V^{\prime}$. It is clear that $U_{(r)} \rightarrow V$ is an isomorphism. The following lemma will complete the proof of the proposition.

LEMma (4.3.1). Suppose $Y$ proper flat over $S, V$ an open subsel of $Y$, $s \in S$. If $V_{s}$ is dense in $Y_{s}$, then there exists an open subset $S_{0} \subset S$ such that for each $s^{\prime} \in S_{0}, V_{s^{\prime}}$ is dense in $Y_{s^{\prime}}$. 
$F=Y \backslash V$ is a closed subset of $Y$. Denote the restriction of $p_{Y}: F \rightarrow$ $S$ by $p^{\prime}$. We define $d^{\circ}$ and $d^{\prime}$ by

$$
\begin{aligned}
& d^{\circ}: Y \longrightarrow Z, y \longmapsto \operatorname{dim}_{y}\left(p_{Y}^{-1}\left(p_{Y}(y)\right),\right. \\
& d^{\prime}: F \longrightarrow Z, \quad y \longmapsto \operatorname{dim}_{y}\left(p^{\prime-1}\left(p^{\prime}(y)\right) .\right.
\end{aligned}
$$

Then $d^{\prime}$ is upper semi-continuous, and $d^{\circ}$ is continuous since $Y$ is flat over $S$. The restriction $d$ of $d^{\circ}$ to $F$ is also continuous. Clearly we have $d(y) \geq d^{\prime}(y)$ for $y \in F$. The denseness of $V_{s}$ in $Y_{s}$ is equivalent to $d(y)>$ $d^{\prime}(y)$ for all $y \in F_{s}$. By the continuity of $d^{\circ}$ and the upper semi-continuity of $d^{\prime}$, for each $y \in F_{s}$, there exists an open neighbourhood $N(y)$ of $y$ in $F$ such that $d\left(y^{\prime}\right)>d^{\prime}\left(y^{\prime}\right)$ if $y^{\prime} \in N(y)$. If $O=\bigcup_{y \in F_{s}} N(y), O$ is open in $F$ with $F_{s} \subset O \subset F$ and $d\left(y^{\prime}\right)>d^{\prime}\left(y^{\prime}\right)$ for $y^{\prime} \in O$. By the properness of $p_{Y}$, $p_{Y}(F \backslash O)$ is closed. We have on the other hand $s \notin p_{Y}(F \backslash O)$ by $F_{s} \subset O$. Let $S_{0}$ be the open subset $S \backslash p_{Y}(F \backslash O)$ in $S$. Then $s \in S_{0}$. If $s^{\prime} \in S_{0}$ and if $y \in F_{s^{\prime}}$, then $y \in O$ so that $d(y)>d^{\prime}(y)$, hence $V_{s^{\prime}}$ is dense in $Y_{s^{\prime}}$.

(4.4) Given a locally noetherian scheme $S$, and $S$-schemes $X$ and $Y$, we shall put

$$
\operatorname{Hom}_{S-\mathrm{rat}}(X, Y)=\Gamma_{S-\mathrm{rat}}(X \underset{S}{\times} Y \mid X),
$$

i.e., the set of closed subschemes $U$ of $X \times \underset{S}{Y}$, flat over $S$ such that $U_{s}$ is geometrically reduced for $s \in S$ and such that there exists an open subset $V$ of $X$ satisfying the conditions 1$) U_{(V)} \rightarrow V$ is an isomorphism, 2) $V_{s}$ is dense in $X_{s}$ for $s \in S$ and 3$)\left(U_{(V)}\right)_{s}$ is dense in $U_{s}$ for $s \in S$. We denote further $\Gamma_{S-\text { rat }}(X \underset{S}{\times} Y \mid X)$ by

$$
\operatorname{Hom}_{S-\mathrm{rat}}(X, Y) \text {. }
$$

For a geometric point $\bar{s}$ of $S$, if $X_{\bar{s}}$ is reduced,

$$
\begin{aligned}
\operatorname{Hom}_{S-\mathrm{rat}}(X, Y)(\bar{s}) & =\Gamma_{(\bar{s})-\mathrm{rat}}\left(X_{\bar{s}} \times \underset{\kappa(\bar{s})}{Y_{\bar{s}}} / X_{\bar{s}}\right) \\
& =\text { the set of } \kappa(\bar{s}) \text {-rational maps from } X_{\bar{s}} \text { to } Y_{\bar{s}} .
\end{aligned}
$$

The proposition (4.3) immediately implies the

Proposition (4.5). Let $S$ be a locally noetherian scheme, $Y$ a projective scheme over $S$, and $X$ a flat projective scheme over $S$. Then the functor $\operatorname{Hom}_{S \text {-rat }}(X, Y)$ is representable by open subscheme $\operatorname{Hom}_{S \text {-rat }}(X, Y)$ of $\operatorname{Hilb}_{X \times Y / S}$.

(4.6) Given an $S$-morphism $f: Z \rightarrow X$, we define 


$$
\operatorname{Hom}_{S-\mathrm{rat}}(Z, f ; X, Y)
$$

as the set of pairs $(\varphi, U)$ of $\varphi \in \operatorname{Hom}_{S}(S, Z)$ and a closed subscheme $U$ of $X \times Y$ flat over $S$ such that $U_{s}$ is geometrically reduced for $s \in S$ and such that there exists an open subscheme $V$ of $X$ satisfying the conditions:

(4.6.1) the morphism $U_{(V)} \rightarrow V$ induced from $U \subset X \underset{S}{\times} Y \rightarrow X$ is an isomorphism;

(4.6.2) $V_{s}$ is dense in $X_{s}$ for $s \in S$;

(4.6.3) $\left(U_{(V)}\right)_{s}$ is dense in $U_{s}$ for $s \in S$;

(4.6.4) $f \circ \varphi(S) \subset V$.

Note that the condition (4.6.4) is the same as $(f \circ \varphi)^{-1}(V)=S$. The set $\operatorname{Hom}_{S \text {-rat }}(Z, f ; X, Y)$ is a subset of $\operatorname{Hom}_{S}(S, Z) \times \operatorname{Hom}_{S-\mathrm{rat}}(X, Y)$. For a morphism $T \rightarrow S$, the correspondence $(\varphi, U) \mapsto\left(\varphi_{T}, U_{T}\right)$ defines a map

$$
\operatorname{Hom}_{S-\mathrm{rat}}(Z, f ; X, Y) \longrightarrow \operatorname{Hom}_{T-\mathrm{rat}}\left(Z_{T}, f_{T} ; X_{T}, Y_{T}\right)
$$

and a contravarient functor

(4.6.5) $\operatorname{Hom}_{S-\mathrm{rat}}(Z, f ; X, Y)$ : (loc. noetherian schemes $\left./ S\right) \longrightarrow($ Sets)

Clearly this is a subfunctor of $h_{Z} \times \operatorname{Hom}_{S-\mathrm{rat}}(X, Y)$. For a geometric point $\bar{s}$ of $S$, if $X_{\bar{s}}$ is reduced, with the above identification,

$$
\begin{aligned}
& \operatorname{Hom}_{S-\text { rat }}(Z, f ; X, Y)(\bar{s})
\end{aligned}
$$

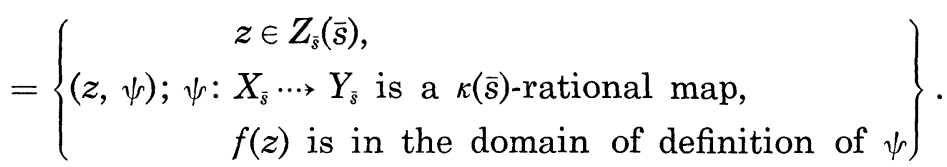

Proposition (4.7). Let $S, X$ and $Y$ be as in (4.5) and $f: Z \rightarrow X$ be an $S$-morphism. Then the functor $\operatorname{Hom}_{S \text {-rat }}(Z, f ; X, Y)$ is representable by an open subscheme $\operatorname{Hom}_{S \text {-rat }}(Z, f ; X, Y)$ of $Z \underset{S}{\times H_{S o m} \text { rat }}(X, Y)$.

It is sufficient to show that if $s \in S$ and if $(\varphi, U) \in \operatorname{Hom}_{S}(S, Z) \times$ $\operatorname{Hom}_{S-\text { rat }}(X, Y)$ is such that $\left(\varphi_{s}, U_{s}\right) \in \operatorname{Hom}_{s-\text { rat }}\left(Z_{s}, f_{s} ; X_{s}, Y_{s}\right)$, then there exists an open neighbourhood $S_{0}$ of the point $s$ in $S$ such that, for $s^{\prime} \in S_{0}$, $\left(\varphi_{s^{\prime}}, U_{s^{\prime}}\right) \in \operatorname{Hom}_{s^{\prime} \text { rat }}\left(Z_{s}, f_{s^{\prime}} ; X_{s^{\prime}}, Y_{s^{\prime}}\right)$, where $s=\operatorname{Spec}(\kappa(s))$ and $s^{\prime}=\operatorname{Spec}\left(k\left(s^{\prime}\right)\right)$. If $V^{\prime}$ is an open subset of $X_{s}$, satisfying the conditions (4.6.1) 4)) (where we replace $S$ and $V$ by $s$ and $V^{\prime}$, respectively), then by the proof of (4.3), there exist an open neighbourhood $S_{1}$ of $s$ in $S$, and an open subscheme $V_{1}$ of $X_{S_{1}}$ satisfying the conditions $\left.(4.6,1) \sim 3\right)$ ) such that $\left(V_{1}\right)_{s}=V^{\prime}$. Then $S_{0}=(f \circ \varphi)^{-1}\left(V_{1}\right)$ is the required open subset. 
(4.8). Suppose $(\varphi, U) \in \operatorname{Hom}_{S-\mathrm{rat}}(Z, f ; X, Y)$. With the above notations, $f \circ \varphi: S \rightarrow X$ is factorized as $S \rightarrow V \subset X$. If $p^{\prime \prime}: U_{(V)} \simeq V$ is induced from $U \subset X \times \underset{S}{\times} Y \rightarrow X$ by the base change $V \rightarrow X$, then we obtain a morphism

$$
\Psi: S \longrightarrow V \stackrel{p^{\prime \prime-1}}{\longrightarrow} U_{(V)} \subset X \underset{S}{\longrightarrow} Y \longrightarrow Y .
$$

Thus we get a map

$$
\operatorname{Hom}_{S-\mathrm{rat}}(Z, f ; X, Y) \longrightarrow \operatorname{Hom}_{S}(S, Y),(\varphi, U) \longmapsto \Psi,
$$

and a collection of this kind of maps defines a morphism of functors

$$
\text { ev: } \operatorname{Hom}_{S-\mathrm{rat}}(Z, f ; X, Y) \longrightarrow \boldsymbol{h}_{Y} \text {. }
$$

In particular, if the hypothesis of (4.7) is satisfied, we obtain a morphism of schemes ev: $H_{o m}$-rat $(Z, f ; C, Y) \rightarrow Y$.

If $(z, \psi) \in \operatorname{Hom}_{S-\text { rat }}(Z, f ; X, Y)(\bar{s})$, where $\bar{s}$ is a geometric point of $S$, and if $X_{\bar{s}}$ is reduced, then $z \in Z_{\bar{s}}(\bar{s})$ and $\psi: X_{\bar{s}} \ldots, Y_{\bar{s}}$ is a rational map with $f(z) \in \operatorname{dom} \psi$, and ev $((z, \psi))=\psi(f(z))$.

$\S 5$.

(5.1) Let $V$ be a smooth projective variety of dimension $m$ over an algebraically closed field $k$ of characteristic zero, and $p$ an integer $\geq 0$. For integers $d, \ell>0$, we put

$$
E_{\ell}^{p}(V)_{d}=\left\{(X, Y) \in C_{r}(V)_{d} \times C_{r}(V)_{d} ; X_{(\ell)} Y \text { on } V\right\}
$$

where $r=m-p$, and we identify the cycles on $V$ with their Chow points. We shall prove the analogues of Propositions (2.2), (2.3) and (2.5).

(5.2) There exists a countable number of families of smooth irreducible projective curve $\mathscr{F}_{\mu} / \mathscr{S}_{\mu}(\mu \in M)$ such that for any smooth irreducible projective curve $C$, there exist a $\mu \in M$ and a point $s \in \mathscr{S}_{\mu}$ such that $C \simeq$ $\left(\mathscr{F}_{\mu}\right)_{s}$; the indexing set $M$ is countable and $\mathscr{F}_{\mu} \rightarrow \mathscr{S}_{\mu}$ is smooth projective and each fibre is irreducible of dimension 1 . Note that the set of $\left(Q, \mu_{1}, \cdots, \mu_{\ell}, d^{\prime}, d^{\prime \prime}\right)$ formed of $Q \in Q[T], \mu_{1}, \cdots, \mu_{\ell} \in M$ and of positive integers $d^{\prime}, d^{\prime \prime}$ is countable.

(5.3) Fix for a moment $\mu_{1}, \cdots, \mu_{\ell} \in M$ and $Q \in Q[T]$, and denote the set of maps from $[1, \ell]$ to $\{0,1\}$ by $2^{[1, \ell]}$, where $[1, \ell]$ is the set of integers between 1 and $\ell$. To simplify the notations, we shall write: 


$$
\begin{gathered}
\mathscr{F}_{i}=\mathscr{F}_{\mu_{i}}, \quad \mathscr{S}_{i}=\mathscr{S}_{\mu_{i}}, \\
\mathscr{F}=\mathscr{F}_{1} \times \cdots \times \mathscr{F}_{\ell}, \\
\mathscr{S}=\mathscr{S}_{1} \times \cdots \times \mathscr{S}_{\ell}
\end{gathered}
$$

Then, $\mathscr{F}$ is a scheme smooth projective over $\mathscr{S}$. For $\sigma \in 2^{[1, \ell]}$, we shall define the $\mathscr{S}$-morphism

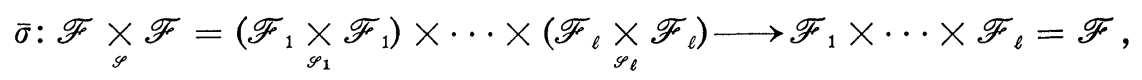

by

$$
\left(x_{1}^{(0)}, x_{1}^{(1)}, \cdots, x_{\ell}^{(0)}, x_{\ell}^{(1)}\right) \longmapsto\left(x_{\ell}^{(\sigma(1))}, \cdots, x_{1}^{(\sigma(\ell))}\right) .
$$

Recall that $\operatorname{Hom}_{\mathscr{S} \text {-rat }}^{Q}\left(\mathscr{F} \underset{\mathscr{F}}{\times} \mathscr{F}, \bar{\sigma} ; \mathscr{F}, \mathscr{S} \times C_{r}(V)_{d^{\prime}} \times C_{r}(V)_{d^{\prime \prime}}\right)$ is an open subscheme of the quasi-projective sheme $(\mathscr{F} \underset{\mathscr{S}}{\times} \mathscr{F}) \times \operatorname{Hom}_{\mathscr{S}-\mathrm{rat}}\left(\mathscr{F}, \mathscr{S} \times C_{r}(V)_{d^{\prime}} \times\right.$ $\left.C_{r}(V)_{d^{\prime \prime}}\right)$, where $Q \in \boldsymbol{Q}[T]$ is the corresponding Hilbert polynomial. Consider

$$
W^{\prime}=\bigcap_{\sigma \in[[1, \ell]} H_{o m}^{Q} Q-\mathrm{rat}\left(\mathscr{F} \underset{\mathscr{S}}{\times} \mathscr{F}, \bar{\sigma} ; \mathscr{F}, \mathscr{S} \times C_{r}(V)_{d^{\prime}} \times C_{r}(V)_{d^{\prime \prime}}\right),
$$

an open subscheme of $(\mathscr{F} \times \mathscr{F}) \times \operatorname{Hom}_{\mathscr{S}-\mathrm{rat}}\left(\mathscr{F}, \mathscr{S} \times C_{r}(V)_{d^{\prime}} \times C_{r}(V)_{d^{\prime \prime}} \quad\right.$ For $\sigma \in 2^{[1, \ell]}$, we have a morphism

$$
\begin{aligned}
\sigma_{*}: W^{\prime} & \subset H m_{\mathscr{f}-\mathrm{rat}}^{Q}\left(\mathscr{F} \underset{\mathscr{S}}{\times} \mathscr{F}, \bar{\sigma} ; \mathscr{F}, \mathscr{S} \times C_{r}(V)_{d^{\prime}} \times C_{r}(V)_{d^{\prime \prime}}\right) \\
& \stackrel{\mathrm{ev}}{\longrightarrow} \mathscr{S} \times C_{r}(V)_{d^{\prime}} \times C_{r}(V)_{d^{\prime \prime}} \stackrel{\text { proj }}{\longrightarrow} C_{r}(V)_{d^{\prime}} \times C_{r}(V)_{d^{\prime \prime}} .
\end{aligned}
$$

For $\sigma \in 2^{[1, \ell]}$, we put $|\sigma|=\sum_{1 \leq i \leq \ell} \sigma(i)$. We define the morphism

$$
\Sigma^{\prime}: W^{\prime} \longrightarrow C_{r}(V)_{N} \times C_{r}(V)_{N}, \quad N=2^{\ell-1}\left(d^{\prime}+d^{\prime \prime}\right),
$$

by

$$
\Sigma^{\prime}(x)=\left(\sum_{|\sigma| \equiv 0} \operatorname{pr}_{1} \circ \sigma_{*}(x)+\sum_{|\sigma| \equiv 1} \operatorname{pr}_{2} \circ \sigma_{*}(x), \sum_{|\sigma| \equiv 1} \operatorname{pr}_{1} \circ \sigma_{*}(x)+\sum_{|\sigma| \equiv 0} \mathrm{pr}_{2} \circ \sigma_{*}(x)\right),
$$

where $\equiv$ means "modulo 2" and "t" are the morphisms of the form

$$
C_{r}(V)_{d} \times C_{r}(V)_{d^{\prime}} \longrightarrow C_{r}(V)_{d+d^{\prime}}, \quad(X, Y) \longmapsto X+Y,
$$

and $\mathrm{pr}_{1}, \mathrm{pr}_{2}$ are the projections on $C_{r}(V)_{d^{\prime}}$ and $C_{r}(V)_{d^{\prime \prime}}$. Finally consider the morphism

$$
\begin{aligned}
\sum: C_{r}(V)_{d} \times C_{r}(V) \times W^{\prime} \longrightarrow C_{r}(V)_{d+N} \times C_{r}(V)_{d+N}, & \underset{\Psi}{\Psi} \\
(X, Y, x) & \longmapsto \Phi\left((X, Y), \Sigma^{\prime}(x)\right)
\end{aligned}
$$


where $\Phi: C_{r}(V)_{d} \times C_{r}(V)_{d} \times C_{r}(V)_{N} \times C_{r}(V)_{N} \rightarrow C_{r}(V)_{d+N} \times C_{r}(V)_{d+N}$ is such that $\left(X, Y, X^{\prime}, Y^{\prime}\right) \mapsto\left(X+X^{\prime}, Y+Y^{\prime}\right)$. We put $W=\Sigma^{-1}(\Delta), \Delta$ being the diagonal of $C_{r}(V)_{d+N} \times C_{r}(V)_{d+N}$. We shall then set

$$
E_{l}^{p}(V)_{d}^{\left(Q, \mu_{1}, \cdots, \mu_{\ell}, d^{\prime}, d^{\prime \prime}\right)}=\operatorname{pr} W,
$$

where pr: $C_{r}(V)_{d} \times C_{r}(V)_{d} \times W^{\prime} \rightarrow C_{r}(V)_{d} \times C_{r}(V)_{d}$ is the projection; hence pr $W$ is a constructible subset of $C_{r}(V)_{d} \times C_{r}(V)_{d}$.

(5.4) Let $\varphi: S \rightarrow W$, i.e., $\varphi \in W(S)$. Since $W \subset C_{r}(V)_{d} \times C_{r}(V)_{d} \times W^{\prime}$, $\varphi$ is of the form $\varphi=(f, g, h) \in C_{r}(V)_{d}(S) \times C_{r}(V)_{d}(S) \times W^{\prime}(S)$. Note that

$$
\mathscr{F}_{S}=\mathscr{F} \underset{\mathscr{S}}{\times} S=\left(\mathscr{F}_{1} \times \cdots \times \mathscr{F}_{\ell}\right)_{S_{1} \times \cdots \times \mathscr{S}_{q}} S=\left(\mathscr{F}_{1}\right)_{S} \underset{S}{\times} \cdots \underset{S}{\times}\left(\mathscr{F}_{\ell}\right)_{S},
$$

where $\left(\mathscr{F}_{i}\right)_{S}=\mathscr{F}_{i} \times \underset{s}{\times} S$. By definition,

$$
\begin{aligned}
& W^{\prime}(S)=\bigcap_{\sigma} H_{o m}^{Q} m_{\mathscr{s} \text {-at }}^{Q}\left(\mathscr{F} \underset{\mathscr{S}}{\times} \mathscr{F}, \bar{\sigma} ; \mathscr{F}, \mathscr{S} \times C_{r}(V)_{d^{\prime}} \times C_{r}(V)_{d^{\prime \prime}}\right)(S) \\
& =\bigcap_{o} \operatorname{Hom}_{S-\text { rat }}^{Q}\left(\mathscr{F}_{S} \underset{S}{\times} \mathscr{F}_{S}, \bar{\sigma}_{S} ; \mathscr{F}_{S}, S \times C_{r}(V)_{d^{\prime}} \times C_{r}(V)_{d^{\prime \prime}}\right) \\
& \subset\left(\mathscr{F}_{S} \underset{S}{\times \mathscr{F}_{S}}\right)(S) \times \operatorname{Hom}_{S-\text { rat }}^{Q}\left(\mathscr{F}_{S}, S \times C_{r}(V)_{d^{\prime}} \times C_{r}(V)_{d^{\prime \prime}}\right), \\
& \left(\mathscr{F}_{S} \underset{S}{\times \mathscr{F}_{S}}\right)(S)=\mathscr{F}_{1}(S) \times \mathscr{F}_{1}(S) \times \cdots \times \mathscr{F}_{\ell}(S) \times \mathscr{F}_{\ell}(S) .
\end{aligned}
$$

So $h=\left(s_{1}^{(0)}, s_{1}^{(1)}, \cdots, s_{\ell}^{(0)}, s_{\ell}^{(1)}, \bar{\psi}\right)$ for $s_{\imath}^{(j)} \in \mathscr{F}_{i}(S), j=0,1 ; i=0, \cdots, \ell$, and for $\bar{\psi} \in \operatorname{Hom}_{S-\text { rat }}^{Q}\left(\mathscr{F}_{S}, S \times C_{r}(V)_{d^{\prime}} \times C_{r}(V)_{d^{\prime \prime}}\right)$. Then $\bar{\psi}$ defines a rational map

$$
\psi: \mathscr{F}_{S} \ldots \ldots \rightarrow C_{r}(V)_{d^{\prime}} \times C_{r}(V)_{d^{\prime \prime}} .
$$

By definition, $\psi \circ s^{\sigma}$ are defined for any $\sigma \in 2^{[1, \ell]}$, where

$$
s^{\sigma}=s^{(\sigma(1))} \times \cdots \times s^{(\sigma(g))} \in\left(\left(\mathscr{F}_{1}\right)_{S} \underset{S}{\times} \cdots \underset{S}{\times}\left(\mathscr{F}_{\ell}\right)_{S}\right)(S) .
$$

The definition of $W$ implies that

$$
\begin{aligned}
& f+\sum_{|\sigma| \equiv 0} \operatorname{pr}_{1} \circ \psi \circ s^{\sigma}+\sum_{|\sigma| \equiv 1} \operatorname{pr}_{2} \circ \psi \circ s^{\sigma} \\
& \quad=g+\sum_{|\sigma| \equiv \equiv} \operatorname{pr}_{1} \circ \psi \circ s^{\sigma}+\sum_{|\sigma| \equiv 0} \operatorname{pr}_{2} \circ \psi \circ s^{\sigma} .
\end{aligned}
$$

in $C_{r}(V)_{d+N}(S)$.

Conversely, suppose $S=$ Spec $k$ and suppose that $\mu_{1}, \cdots, \mu_{c} \in M$, $S \rightarrow \mathscr{S}_{\mu_{i}}$ are given. Hence $\mathscr{F}_{4}$ can be defined as above. Let $f, g \in C_{r}(V)_{d}(S)$, and $\psi=\bar{\psi}: \mathscr{F}_{S} \cdots C_{r}(V)_{d^{\prime}} \times C_{r}(V)_{d^{\prime \prime}}=S \times C_{l}(V)_{d^{\prime}} \times C_{r}(V)_{d^{\prime \prime}}$ be a rational map. If $s_{i}^{(j)} \in \mathscr{F}_{i}(S)$ satisfy the conditions $\operatorname{Im} s^{\sigma} \subset$ dom $\psi$ for $\sigma \in 2^{[1, \ell]}$, and if the equality (5.4.1) holds, then $\varphi=(f, g, h) \in W(S), h=\left(s_{1}^{(0)}, s_{1}^{(1)}, \cdots, s_{\iota}^{(0)}\right.$, $\left.\boldsymbol{s}_{\ell}^{(1)}, \bar{\psi}\right)$, where $W$ is defined as above for an appropriate $Q \in \boldsymbol{Q}[T]$. 
Theorem (5.5). Under the hypotheses the notations as above, we have:

$$
E_{\ell}^{p}(V)_{d}=\bigcup E_{\ell}^{p}(V)_{d}^{\left(Q, \mu_{1}, \cdots, \mu_{\ell}, d^{\prime}, d^{\prime \prime}\right)} \quad \text { (as closed points), }
$$

where the union is taken over all $Q \in \boldsymbol{Q}[T], \mu_{1}, \cdots, \mu_{\ell} \in M$ and positive integers $d^{\prime}$ and $d^{\prime \prime}$.

$$
E_{\ell}^{p}(V)_{d} \supset E_{\ell}^{\bar{\nu}}(V)_{d}^{\left.\overline{(Q, \mu}, \mu_{1}, \cdots, \mu_{\ell}, d^{\prime}, d^{\prime \prime}\right)}
$$

for arbitrary $Q, \mu_{i}$, and $d^{\prime}$ and $d^{\prime \prime}$, where the bar denotes the closure in $C_{r}(V)_{d} \times C_{r}(V)_{d}$. In particular, $E_{\ell}^{p}(V)_{d}$ is a countable union of closed subsets of $C_{r}(V)_{d} \times C_{r}(V)_{d}$. Moreover, if we replace $\mathscr{F}_{\mu} / \mathscr{S}_{\mu}^{\prime} \mathrm{s}$ by their associated families of jacobians in (5.3), then the equality (5.5.1) also holds.

The equality (5.5.1) follows from $(3.8,1)$ ) and (5.4). To show (5.5.2), notice first that $E_{\ell}^{p}(V)_{d}^{\left(Q, \mu_{1}, \cdots \mu_{\ell}, d^{\prime}, d^{\prime \prime}\right)}=E_{\ell}^{p}(V)_{d}^{\left(Q, \cdots, d^{\prime \prime}\right)}$ is constructible. Hence it is sufficient to verify that if $\Gamma$ is a curve (maybe singular or noncomplete, but irreducible) in $E_{\ell}^{p}(V)_{d}^{\left(Q, \cdots, d^{\prime \prime}\right)}$, then the closure $\bar{\Gamma}$ of $\Gamma$ in $C_{r}(V)_{d} \times C_{r}(V)_{d}$ is in $E_{\ell}^{p}(V)_{d}$. With the notations in (5.3), there exists a curve $\Gamma^{\prime}$ in $W$ dominating $\Gamma$ via the morphism pr. Let $R^{\prime}$ be the smooth projective model of $\Gamma^{\prime}$, and $R_{0}^{\prime}$ the open subvariety of $R^{\prime}$ such that $R_{0}^{\prime}$ is the normalization of $\Gamma^{\prime}: R_{0}^{\prime} \rightarrow \Gamma^{\prime}$. We get the morphisms

$$
\begin{aligned}
R_{0}^{\prime} \longrightarrow & \Gamma^{\prime} \subset W \subset C_{r}(V)_{d} \times C_{r}(V)_{d} \times(\mathscr{F} \times \underset{\mathscr{S}}{\times} \mathscr{F}) \\
& \times{ }_{\mathscr{S}} \operatorname{Hom}_{\mathscr{S} \text {-rat }}\left(\mathscr{F}, \mathscr{S} \times C_{r}(V)_{d^{\prime}} \times C_{r}(V)_{d^{\prime \prime}}\right) \\
& \longrightarrow \mathscr{S}=\mathscr{S}_{1} \times \cdots \times \mathscr{S}_{\ell} \longrightarrow \mathscr{S}_{i}, \quad i=1, \cdots, \ell,
\end{aligned}
$$

and thus the smooth families of curves $\left(\mathscr{F}_{i}\right)_{R_{0}^{\prime}} / R_{0}^{\prime}, i=1, \cdots, \ell$, by the base change $R^{\prime} \rightarrow \mathscr{S}_{i}$. By virtue of [2], (2.7), if the genus of the generic fibre of $\left(\mathscr{F}_{i}\right)_{R_{0}^{\prime}} / R_{0}^{\prime}$ is $\geq 2$, there exist a smooth projective curve $B_{i}$ and a stable curve $F_{i}^{\prime} / B_{i}$ such that $B_{i}$ is a covering of $R^{\prime}$ and $\left(F_{i}^{\prime}\right)_{B_{i}^{0}} \simeq\left(\mathscr{F}_{i}\right)_{B_{i}^{0}}$, where $B_{i}^{0}=B_{i} \underset{R^{\prime}}{\times} R_{0}^{\prime}$. If the generic fibre of $\left(\mathscr{F}_{i}\right)_{R_{0}^{\prime}} / R_{0}^{\prime}$ has genus 1 , by the argument in [2], $\S 2$, there exist a smooth projective curve $B_{i}$ which is a covering of $R^{\prime}$, and a flat family of curves $F_{i}^{\prime} \mid B_{i}$ such that $\left(F_{i}^{\prime}\right)_{B_{i}^{0}} \simeq\left(\mathscr{F}_{i}\right)_{B_{i}^{0}}$ and such that the (geometric) fibres of $F_{i}^{\prime} \mid B_{i}$ are reduced and have at worst ordinary double points. Finally, if the generic fibre is of genus 0 , then there are a smooth projective curve $B_{i}$ which is a covering of $R^{\prime}$, and a flat family of curves $F_{i}^{\prime} / B_{i}$ with fibres $P_{1}$ such that $\left(F_{i}^{\prime}\right)_{B_{i}^{0}} \simeq\left(\mathscr{F}_{i}\right)_{B_{i}^{0}}$. Let $R$ be a smooth projective curve with $R \rightarrow B_{i}, i=1, \cdots, \ell$, such that the morphisms $R \rightarrow B_{i} \rightarrow R^{\prime}$ are the same. The fibres of $F_{i}^{\prime} \underset{B_{i}}{\times} R=\left(F_{i}^{\prime}\right)_{R} / R$ are reduced 
and have at worst ordinary double points and $\left(F_{\imath}^{\prime}\right)_{R} \underset{R}{\times} R_{0} \simeq\left(\mathscr{F}_{i}\right)_{R_{0}^{\prime}} \underset{R_{0}^{\prime}}{\times} R_{0}$, where $R_{0}=R \times R_{R^{\prime}}$. Blowing up $\left(F_{\imath}^{\prime}\right)_{R}$ at points over $R \backslash R_{0}$ if necessary, we obtain flat families of curves $F_{i} / R$ such that $F_{i}$ are smooth projective surfaces and $\left(F_{i}\right)_{R_{0}} \simeq\left(\mathscr{F}_{i}\right)_{R_{0}^{\prime}} \underset{R_{0}^{\prime}}{\times} R_{0}$ and such that the fibres of $F_{\imath} / R$ are reduced and have at worst ordinary double points. We put $F=F_{1} \times \underset{R}{ } \cdots$ $\underset{R}{\times} F_{\ell}$. Considering $R_{0} \rightarrow R_{0}^{\prime} \rightarrow W$, we are in the situation remarked in (5.4): we have $R_{0}$-sections $s_{i}^{(j)}: R_{0} \rightarrow\left(F_{i}\right)_{R_{0}}, j=0,1 ; i=1, \cdots, \ell$, a rational map

$$
\psi: F_{R_{0}} \ldots \ldots \rightarrow C_{r}(V)_{d^{\prime}} \times C_{\imath}(V)_{d^{\prime \prime}},
$$

and morphisms $f: R_{0} \rightarrow C_{r}(V)_{d}$ and $g: R_{0} \rightarrow C_{r}(V)_{d}$ satisfying the equality (5.4.1) in which

$$
s^{\sigma}=s_{1}^{(\sigma(1))} \times \cdots \times s_{\ell}^{(\sigma(\ell))}: R_{0} \longrightarrow F_{R_{0}}=\left(F_{1}\right)_{R_{0}} \underset{R_{0}}{\times} \cdots \underset{R_{0}}{\times}\left(F_{\ell}\right)_{R_{0}},
$$

and $\operatorname{Im} s^{\sigma} \subset \operatorname{dom} \psi$. Since $R$ is a smooth curve, and $F_{i} / R$ is proper, the $R_{0}$-sections $s_{i}^{(j)}$ have their extensions $\overline{\boldsymbol{s}}_{i}^{(j)}: R \rightarrow F_{i}$. Moreover $f, g: R_{0} \rightarrow$ $C_{r}(V)_{d}$ are extended to $\bar{f}, \bar{g}: R \rightarrow C_{r}(V)_{d}$ by the completeness of $C_{r}(V)_{d}$. Note that $F$ is integral because $F / R$ is flat and the generic fibre of $F / R$ is integral. Since $F_{R_{0}}$ is an open subset of $F, \psi$ defines a rational map $\bar{\psi}: F \ldots C_{r}(V)_{d^{\prime}} \times C_{r}(V)_{d^{\prime \prime}}$. Defining $\bar{s}^{\sigma}$ as $s^{\sigma}$, $\bar{\psi} \circ \bar{s}^{\sigma}$ are morphisms and the equality (5.4.1) holds replacing $f, g, \psi$ and $s^{\sigma}$ by $\bar{f}, \bar{g}$, $\bar{\psi}$ and $\bar{s}^{\sigma}$, respectively. To show $\bar{\Gamma} \in E_{\ell}^{p}(V)_{d}$, it suffices to prove that for any $r \in R$, we have $\bar{f}(r)_{(\varepsilon)} \bar{g}(r)$. But by the extended version of the equality (5.4.1), it is sufficient to verify

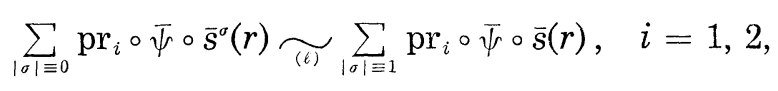

for any $r \in R$. To simplify the notations, replacing $\mathrm{pr}_{i} \circ \bar{\psi}$ by $\bar{\psi}$, we can assume $\bar{\psi}: F \ldots C_{r}(V)_{d^{\prime}}$. Let $r \in R$ be a point such that some of $\left(F_{\imath}\right)_{r}$ $(i=1, \cdots, \ell)$ have singularities. We notice that the set of such $r$ 's is a finite set. For $j, 1 \leq j \leq \ell$, let $L_{j}$ be the set of points $x \in F_{r}=\left(F_{1}\right)_{r} \times \cdots$ $\times\left(F_{\ell}\right)_{r}$ at least $j$ of whose projections onto $\left(F_{i}\right)_{r}$ 's fall to double points. Then $L_{1} \supset L_{2} \supset \cdots \supset L_{\ell}$ are closed subsets of $F_{r}$, hence of $F$. We endow the reduced subscheme structures to $L_{j}$ 's. Blow up first $F$ with center $L_{\ell}$ to obtain $F^{\prime}$ and let $L_{1}^{\prime} \supset L_{2}^{\prime} \supset \ldots \supset L_{\ell-1}^{\prime}$ be the strict transforms of $L_{1} \supset \cdots \supset L_{\ell-1}$; then blow up $F^{\prime}$ with center $L_{\ell-1}^{\prime}$ to obtain $F^{\prime \prime}$ and so on until we blow up $F$ virtually with center $L_{2}$ to obtain $\tilde{F}$ and a morphism 
$\tilde{F} \rightarrow F$. We assert:

I) $\tilde{F}$ is non-singular at the points of the fibre over $r \in R$.

II) For each $x \in F$, any two points of the fibre $\tilde{F}_{x}$ are joined by a finite numbers of rational curves in the fibre.

To show these assertions, we shall recall basic facts about the blowingups: Let $X$ be a scheme, $Y \supset Z$ be its closed subschemes, $X_{1}$ the blowingup of $X$ with center $Z$ and $Y$ the strict transform of $Y$. If $X^{\prime} \rightarrow X$ is a flat morphism, setting $Y^{\prime}=Y_{\left(X^{\prime}\right)}$ and $Z^{\prime}=Z_{\left(X^{\prime}\right)}, X \underset{X}{\times} X_{1}$ is the blowing-up of $X^{\prime}$ with center $Z^{\prime}$, and $X^{\prime} \underset{X}{\times} Y_{1}$ is the strict transform of $Y^{\prime}$. Pick up a point $x \in F$ and put $\hat{\Phi}=\operatorname{Spec}\left(\hat{\mathcal{O}}_{F, x}\right)$ where $\hat{\mathcal{O}}_{F, x}$ is the completion of $\mathcal{O}_{F, x}$ with respect to the maximal ideal. Then we have a flat morphism $\hat{\Phi} \rightarrow F$. Denote by $x_{i}$ the image of $x \in F$ by the projection $F \rightarrow F_{i}$. Rearranging the numbers of indices, we can assume that $x_{1}, \cdots, x_{\ell^{\prime}}$ are ordinary double points and $x_{\ell^{\prime}+1}, \cdots, x_{\ell}$ are smooth points. Hence we have $x \in L_{\ell^{\prime}}$ but $x \notin L_{\ell^{\prime}+1}$. Since $F_{i}$ are smooth,

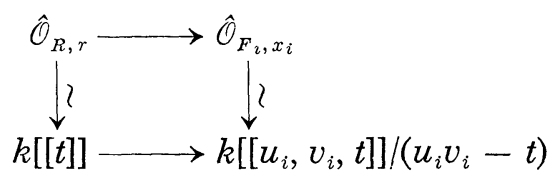

for $i \leq \ell^{\prime}$ and

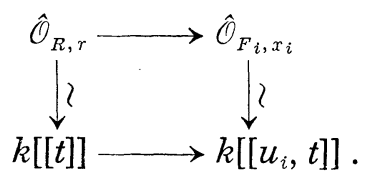

for $i>\ell^{\prime}$. Also we have

$$
\begin{aligned}
\hat{\mathcal{O}}_{F, x} & =\left(\hat{\mathcal{O}}_{F_{1}, x_{1}} \otimes \cdots \otimes_{\hat{\mathcal{O}}_{R, r}} \cdots \hat{\mathcal{O}}_{F, x}\right)^{\wedge} \\
& =k\left[\left[u_{1}, v_{1}, \cdots, u_{\ell^{\prime}}, v_{\ell^{\prime}}, u_{\ell^{\prime}+1}, \cdots, u_{\ell}, t\right]\right] /\left(u_{1} v_{1}-t, \cdots, u_{\ell^{\prime}} v_{\ell^{\prime}}-t\right) .
\end{aligned}
$$

The ideal of $\hat{\mathcal{O}}_{F, x}$ defining $\left(L_{i}\right)_{\hat{\Phi}}=\hat{L}_{i}$ is

$$
\bigcap_{\lambda_{1}, \cdots, \lambda_{i}}\left(u_{\lambda_{1}}, v_{\lambda_{1}}, \cdots, u_{\lambda_{i}}, v_{\lambda_{i}}\right) \text {, }
$$

where the intersection is over those $\left(\lambda_{1}, \cdots, \lambda_{i}\right)$ that satisfy $1 \leq \lambda_{1}<\cdots$ $<\lambda_{i} \leq \ell^{\prime}$. As we have remarked above, $\tilde{F}_{\hat{\phi}}$ is obtained by the same procedure as $\tilde{F}$ replacing $F$ by $F_{\hat{\phi}}$ and $L_{i}$ 's by $\hat{L}_{i}$ 's. We note that the following are equivalent: 
- $F$ is smooth at the points of the fibre over $x$;

- $\tilde{F}_{\hat{\phi}}$ is regular at the closed points of the fibre over the unique closed point $\bar{x}$ of $\hat{\Phi}$.

Moreover, we have $\tilde{F}_{x} \simeq\left(\tilde{F}_{\hat{\phi}}\right)_{\tilde{x}}$. Starting with

$$
\Phi=\operatorname{Spec}\left(k\left[\left[u_{1}, v_{1}, \cdots, u_{\ell^{\prime}}, v_{\ell^{\prime}}, u_{\ell^{\prime}+1}, \cdots, u_{\ell}, t\right]\right] /\left(u_{1} v_{1}-t, \cdots, u_{\ell^{\prime}} v_{\ell^{\prime}}-t\right)\right)
$$

and $A_{i}$ defined by the ideal of the form (5.5.3) in the affine ring of $\Phi$, we define $\tilde{\Phi}$ by the same process as $\tilde{F}$, replacing $F$ by $\Phi$ and $L_{i}$ 's by $\Lambda_{i}$ 's. We have an obvious flat morphism $\hat{\Phi} \rightarrow \Phi$ and $\hat{L}_{i}=\left(\Lambda_{i}\right)_{\hat{\phi}}$. To verify the assertions I) and II) above, it suffices to show that $\tilde{\Phi}$ is smooth at the points of the fibre over the point $x_{0}$ corresponding to the ideal $\left(u_{1}, v_{1}, \cdots\right.$, $\left.u_{\ell^{\prime}}, v_{\ell^{\prime}}, u_{\ell^{\prime}+1}, \cdots, u_{\ell}, t\right)$ and that $\tilde{\Phi}_{x_{0}}$ has the property described in II), because of $\tilde{\Phi} \underset{\phi}{\times} \widehat{\Phi} \simeq \tilde{F}_{\hat{\phi}}$. We shall prove our assertions by induction on $\ell$, so that we assume our assertions for

$$
\operatorname{Spec}\left(k\left[u_{1}, v_{1}, \cdots, u_{n^{\prime}}, v_{n^{\prime}}, u_{n^{\prime}+1}, \cdots, u_{n}, t\right] /\left(u_{1} v_{1}-t, \cdots, u_{n^{\prime}} v_{n^{\prime}}-t\right)\right)
$$

for $n^{\prime} \leq n \leq \ell$. Considering the smooth base change

$$
\begin{gathered}
\operatorname{Spec}\left(k\left[u_{1}, v_{1}, \cdots, u_{\ell^{\prime}}, v_{\ell^{\prime}}, u_{\ell^{\prime}+1}, \cdots, u_{i}, t\right] /\left(u_{1} v_{1}-t, \cdots, u_{\ell^{\prime}} v_{\ell^{\prime}}-t\right)\right) \\
\longrightarrow \operatorname{Spec}\left(k\left[u_{1}, v_{1}, \cdots, u_{\ell^{\prime}}, v_{\ell^{\prime}}, t\right] /\left(u_{1} v_{1}-t, \cdots, u_{\ell^{\prime}} v_{\ell^{\prime}}-t\right)\right)
\end{gathered}
$$

we may assume $\ell^{\prime}=\ell$.

In $\operatorname{Spec}\left(k\left[u_{1}, v_{1}, \cdots, u_{\ell}, v_{\ell}\right]\right), \Phi$ is defined by

$$
u_{1} v_{1}=\cdots=u_{\ell} v_{\ell}=t \text {. }
$$

Then $A_{\ell}=x_{0}$ is defined by $u_{1}=v_{1}=\cdots=u_{\ell}=v_{\ell}=t=0$. The dlowingup $\Phi^{\prime}$ of $\Phi$ at $A_{b}$ is gluing of affine pieces of the following type: in the coordinate system $u_{1} / u_{\ell}, v_{1} / u_{\ell}, \cdots, u_{\ell-1} / u_{\ell}, v_{\ell-1} / u_{\ell}, u_{\ell}, v_{\ell} / u_{\ell}, t$, $\Phi^{\prime \circ}:$

$$
\left(u_{1} / u_{\ell}\right)\left(v_{1} / u_{\ell}\right)=\cdots=\left(u_{\ell-1} / u_{\ell}\right)\left(v_{\ell-1} / u_{\ell}\right)=v_{\ell} / u_{\ell},
$$

$$
t=u_{\ell}^{2}\left(v_{\ell} / u_{\ell}\right) .
$$

On the other hand, $\Lambda_{\ell-1}$ is the union of

$$
u_{1}=v_{1}=\cdots=u_{i-1}=v_{i-1}=u_{i+1}=v_{i+1}=\cdots=u_{\ell}=v_{\ell}=0,
$$

for $i=1, \cdots, \ell$; hence its strict transform $A_{\ell-1}^{\prime}$ in this piece is

$$
u_{1} / u_{\ell}=v_{1} / u_{\ell}=\cdots=u_{\ell-1} / u_{\ell}=v_{\ell-1} / u_{\ell}=0 .
$$

Moreover the strict transform $A_{i-2}^{\prime}$ in this affine piece is the union of 


$$
\begin{aligned}
u_{1} / u_{\ell}=v_{1} / u_{\ell} & =\cdots=u_{i-1} / u_{\ell}=v_{i-1} / u_{\ell}=u_{i+1} / u_{\ell}=v_{i+1} / u_{\ell} \\
& =\cdots=u_{\ell-1} / u_{\ell}=v_{\ell-1} / u_{\ell}=0
\end{aligned}
$$

for $i=1, \cdots, \ell ; \cdots$; and the strict transform $\Lambda_{2}^{\prime}$ of $\Lambda_{2}$ is, in the piece, given by the union of

$$
u_{i} / u_{\ell}=v_{i} / u_{\ell}=u_{j} / u_{\ell}=v_{j} / u_{\ell}=0,
$$

for $1 \leq i<j<\ell$. Denoting $u_{\imath}^{\prime}=u_{i} / u, v_{i}^{\prime}=v_{i} / u(i=1, \cdots, \ell-1)$ and $t^{\prime}=v_{\ell} / u_{\ell}$, we have

$$
\Phi^{\prime \circ}=\operatorname{Spec}\left(k\left[u_{1}^{\prime}, v_{1}^{\prime}, \cdots, u_{\ell-1}^{\prime}, v_{\ell-1}^{\prime}, t^{\prime}\right] /\left(u_{1}^{\prime} v_{1}^{\prime}-t^{\prime}, \cdots, u_{\ell-1}^{\prime} v_{\ell-1}^{\prime}-t^{\prime}\right)\right),
$$

and the ideal of $\Lambda_{i}^{\prime}$ is of the form (5.5.3), replacing $u_{i}$ and $v_{i}$ by $u_{i}^{\prime}$ and $v_{i}^{\prime}$; hence by inducting hypothesis, we see the first assertion.

We shall verify the second assertion; let $\tilde{x}$ and $\tilde{x}^{\prime}$ be the points of $\tilde{\Phi}$ whose images $x$ and $x^{\prime}$ in $\Phi$ are identical, and let $x_{1}$ and $x_{1}^{\prime}$ be the images of $\tilde{x}$ and $\tilde{x}^{\prime}$ in $\Phi^{\prime}$. If $x_{1}=x_{1}^{\prime}$, then by induction hypothesis, we are done. Suppose $x_{1} \neq x_{1}^{\prime}$. Since the morphism $\Phi^{\prime} \rightarrow \Phi$ is an isomorphism outside $\Lambda_{\ell}$, we have $x=x^{\prime} \in \Lambda_{\ell}$, so that $x_{1}, x_{1}^{\prime} \in \Phi_{\Lambda_{\ell}}^{\prime}$. We claim that there exists a finite number of rational curves $\Gamma_{i}(i=1, \cdots, n)$ in $\Phi_{\Lambda_{\ell}}^{\prime}$ such that $\Gamma_{i} \not \subset \Lambda_{2}^{\prime}$ for all $i, x_{1} \in \Gamma_{1}, x_{1}^{\prime} \in \Gamma_{n}$ and $\Gamma_{i} \cap \Gamma_{i+1} \neq \phi(1 \leq i<n)$; This claim implies our second assertion: the curves $\Gamma_{\imath}$ 's lift to the rational curves $\tilde{\Gamma}_{i}$ in $\tilde{\Phi}$. If $y_{i} \in \Gamma_{i} \cap \Gamma_{i+1}(1 \leq i<n)$, if $\tilde{y}_{i} \in \tilde{\Gamma}_{i}$ and $\bar{y}_{i+1} \in \tilde{\Gamma}_{i+1}$ are points over $y_{i}$ and if $\bar{x}$ and $\bar{x}^{\prime}$ are points on $\tilde{\Gamma}_{1}$ and on $\tilde{\Gamma}_{n}$ over $x_{1}$ and $x_{1}^{\prime}$ respectively, by induction hypothesis, $\tilde{y}_{i}$ and $\bar{y}_{i+1}$ can be joined by a finite number of rational curves and the same is true for $\bar{x}$ and $\tilde{x}$, and also for $\bar{x}^{\prime}$ and $\tilde{x}$. Moreover $\bar{y}_{i}$ and $\tilde{y}_{i}$ are on the curve $\tilde{\Gamma}_{i}$, whence the assertion II). We show the claim for arbitrary $x_{1}, x_{1}^{\prime} \in \Phi^{\prime}$ over $\Lambda_{\ell}$, hence we may assume that $x_{1}$ and $x_{1}^{\prime}$ are contained in the same piece of $\Phi^{\prime}$ above, say, the above piece. We shall denote the varieties intersected with the above affine piece by the same symbols as the varieties. The variety $\Phi_{\Lambda_{\ell}}^{\prime}$ is defined by

$$
\begin{gathered}
\left(u_{1} / u_{\ell}\right)\left(v_{1} / u_{\ell}\right)=\cdots=\left(u_{\ell-1} / u_{\ell}\right)\left(v_{\ell-1} / u_{\ell}\right)=v_{\ell} / u_{\ell} . \\
u_{\ell}=t=0 .
\end{gathered}
$$

We can therefore take $\left(u_{1} / u_{\ell}, v_{1} / u_{\ell}, \cdots, v_{\ell-1} / u_{\ell}, v_{\ell} / u_{\ell}\right)$ as the coordinate system for $\Phi_{\Lambda_{\ell}}^{\prime}$. Let $\left(a_{1}, b_{1}, \cdots, a_{\ell-1}, b_{\ell-1}, c\right)$ and $\left(a_{1}^{\prime}, b_{1}^{\prime}, \cdots, a_{\ell-1}^{\prime}, b_{\ell-1}^{\prime}, c^{\prime}\right)$ be the coordinates of the points $x_{1}$ and $x_{1}^{\prime}$. To show $x_{1}$ and $x_{1}^{\prime}$ joined by a rational curve in $\Phi_{\Lambda_{\ell}}^{\prime}$, it is sufficient to find polynomial functions $f_{1}(w)$, 
$g_{1}(w), \cdots, f_{\ell-1}(w)$ and $g_{\ell-1}(w)$ with $f_{i}(0)=a_{\imath}, g_{i}(0)=b_{i}, f_{\imath}(1)=a_{i}^{\prime}$ and $g_{2}(1)$ $=b_{i}^{\prime}$ such that

$$
f_{i}(w) g_{i}(w)=f_{j}(w) g_{j}(w)
$$

for all $1 \leq i<j<\ell$. Note that a point with $v_{\ell} / u_{\ell} \neq 0$ is not in $\Lambda_{2}^{\prime}$. First we shall assume that $c$ and $c^{\prime}$ are non-zero. Considering the functions $f_{\imath}(w)=a_{i} w$ and $g_{i}(w)=b_{\imath} w, x_{1}$ and the point $(0, \cdots, 0)$ are joined. Similarly $x_{1}^{\prime}$ and the point $(0, \cdots, 0)$ are joined. Hence in this case we are done. We then assume $c=0$. We shall show that $x_{1}$ and a point with $v_{\ell} / u_{\ell} \neq 0$ can be joined by a rational curve. Since $a_{i} b_{i}=c=0$ for $i<\ell$, $a_{i}$ or $b_{i}$ is zero. For simplicity, we suppose (and we can suppose) $a_{1} \cdots a_{\nu}$ $\neq 0, b_{1}=\cdots=b_{\nu}=a_{\nu+1}=b_{\nu+1}=\cdots=b_{\nu-1}=0$. We put

$$
\begin{aligned}
& f_{i}(w)=a_{i}+\left(1-a_{i}\right) w, \\
& g_{i}(w)= \begin{cases}w^{2} \prod_{\substack{1 \leq j \leq \nu \\
j \neq \nu}}\left(a_{j}+\left(1-a_{j}\right) w\right) & \text { if } i \leq \nu \\
w & \text { if } \nu<i<\ell .\end{cases}
\end{aligned}
$$

Thus $x_{1}$ and the point with coordinates $(1, \cdots, 1)$ are joined by a rational curve. Since any two points with $v_{\ell} / u_{\ell} \neq 0$ can be joined by rational curves, this completes the proof of our assertions.

Iterating the above procedure at the fibres containing a singularty of $F$, we get a smooth projective variety, again denoted by $\tilde{F}$ and a morphism $\tilde{F} \rightarrow F$ such that for any $x \in F$, the fibre of $\tilde{F}$ over $x$ has the property II), or that the points of the fibre $\tilde{F}_{x}$ are rationally equivalent on $\tilde{F}$. If $z^{\prime}$ denotes the rational equivalence class of the cycle on $\tilde{F} \times V$ corresponding to the rational map $\tilde{\psi}: \tilde{F} \rightarrow F \stackrel{\bar{\psi}}{\rightarrow} C_{r}(V)_{d^{\prime}}$, then $z^{\prime}\left(a^{\prime}\right)\left(a^{\prime} \in \tilde{F}\right)$ depends only on the image $a$ of $a^{\prime}$ in $F$. Hence we denote the rational equivalence class $z^{\prime}\left(a^{\prime}\right)$ by $z(a)$. We notice that the set of singular points of $F$ is of codimension $\geq 3$ in $F$ and hence the complement of the domain of definition of the rational map $\bar{\psi}: F \cdots C_{r}(V)_{d^{\prime}}$ is of codimension $\geq 2$. Let $r \in R$ be an arbitrary point and $C_{i}^{\prime}$ an irreducible component of $\left(F_{i}\right)_{r}$ for each $1 \leq i \leq \ell$. Then $\left(C_{1}^{\prime} \times \cdots \times C_{\ell}^{\prime}\right) \cap \operatorname{dom} \bar{\psi} \neq \phi$, so that $\bar{\psi}$ determines a rational map $\bar{\psi}^{\prime}: C_{1}^{\prime} \times \cdots \times C_{\imath}^{\prime} \cdots>C_{r}(V)_{d^{\prime}}$. Note that

$$
T=\operatorname{dom} \bar{\psi} \cap \operatorname{Reg} F \cap \operatorname{Reg}\left(C_{1}^{\prime} \times \cdots \times C_{\ell}^{\prime}\right)
$$

is a non-empty open subset of $C_{1}^{\prime} \times \cdots \times C_{\ell}^{\prime}$. Let $a \in C_{1}^{\prime} \times \cdots \times C_{\ell}^{\prime}, \Gamma$ be a smooth complete curve, $b \in \Gamma$ a point and $\varphi: \Gamma \rightarrow C_{1}^{\prime} \times \cdots \times C_{\ell}^{\prime}$ a mor- 
phism such that $\varphi^{-1}(T) \neq \phi$ and $\varphi(b)=a$. Then $\bar{\psi}^{\prime} \circ \varphi$ is a morphism from $\Gamma$ to $C_{r}(V)_{d^{\prime}}$; we have therefore a cycle $\bar{\psi}^{\prime} \circ \varphi(b) \in C_{r}(V)_{d^{\prime}}$. Since $\varphi: \Gamma \rightarrow$ $C_{1}^{\prime} \times \cdots \times C_{\ell}^{\prime} \subset F$ lifts to $\tilde{\varphi}: \Gamma \rightarrow \tilde{F}$, and $\tilde{\psi} \circ \tilde{\varphi}=\bar{\psi}^{\prime} \circ \varphi$, the cycle $\bar{\psi}^{\prime} \circ \varphi(b)$ is in the class $z(a)$ by (1.5). Since $\left(F_{i}\right)_{r}$ is connected for $i=1, \cdots, \ell$, there are points $\bar{s}_{i}^{(0)}=c_{i}(0), \cdots, c_{i}\left(n_{i}\right)=\bar{s}_{i}^{(1)}$ on $\left(F_{i}\right)_{r}$ such that $c_{i}(n)$ and $c_{i}(n+1)$ are on the same irreducible component of $\left(F_{i}\right)_{r}$ for $n=0, \cdots$, $n_{i}-1$.

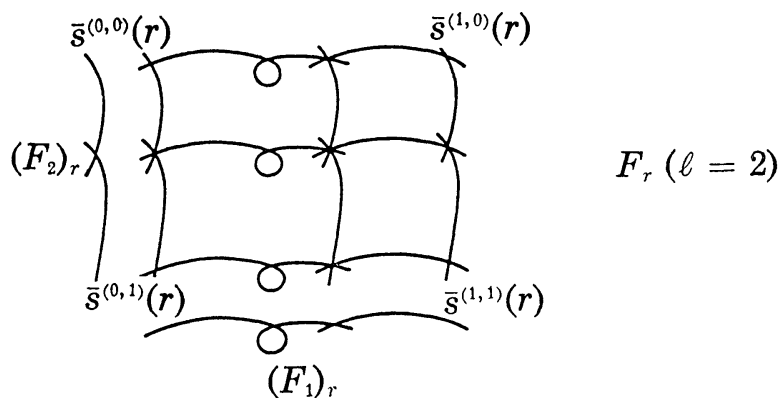

For $\left(j_{1}, \cdots, j_{\ell}\right)\left(0 \leq j_{i} \leq n_{i}\right)$, let $x_{j_{1}, \cdots, j_{\ell}}=z\left(\left(c_{1}\left(j_{1}\right), \cdots, c_{\ell}\left(j_{\ell}\right)\right)\right)$. In virtue of $(3.8,2))$, we have

$$
\sum_{\sigma}(-1)^{|\sigma|} x_{j_{1}+\sigma(1), \ldots, j_{\ell}+\sigma(\ell)} \in F^{\ell} C H^{p}(V) .
$$

On the other hand, $x_{n_{1} \sigma(1), \ldots, n_{\ell} \sigma(\ell)}$ is the class of $\bar{\psi} \circ \bar{s}^{\sigma}(r)$ for any $\sigma \in 2^{[1, \ell]}$ by (1.5). Summing (5.5.4) over $0 \leq j_{i}<n_{i}(i=1, \cdots, \ell)$, we see that the rational equivalence class of $\sum_{\sigma}(-1)^{|\sigma|} \bar{\psi} \circ \bar{s}^{\sigma}(r)$ is in $F^{\ell} C H^{p}(V)$, i.e.,

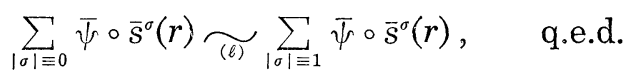

Corollary (5.5.5). If $\kappa: T \rightarrow C H^{p}(V)$ is regular, then for any $\ell>0$, $\kappa^{-1}\left(F^{\ell} \mathrm{CH}^{p}(V)\right)$ is a countable union of closed subsets of $T$.

The proof is similar to that of (2.7).

From now on, we suppose that $k$ is uncountable.

THEOREM (5.6). Let $V$ be a smooth projective variety, $T$ a smooth quasiprojective variety, $f$ and $g: T \rightarrow C_{r}(V)_{d}$ morphisms such that for $t \in T$, $f(t) \underset{(i)}{\sigma} g(t)$. Then there exist a smooth quasi-projeciive variety $S$, families $\Gamma_{i} / S$ of curves (resp. jacobian varieties) over $S$ (each fibre is a smooth projective curve (resp. jacobian variety)), sections $s_{i}^{(0)}, s_{i}^{(1)}: S \rightarrow \Gamma_{i}(1 \leq i \leq \ell)$ of $\Gamma_{i} / S$, a dominant morphism $e: S \rightarrow T$, and a rational map 


$$
H: \Gamma_{1} \underset{S}{\times} \cdots \underset{S}{\times} \Gamma_{\ell} \cdots \cdots, C_{r}(V)_{d^{\prime}} \times C_{r}(V)_{d^{\prime \prime}}
$$

$\left(d^{\prime}, d^{\prime \prime}>0\right)$ such that

$$
\begin{aligned}
& H \text { is defined on } s^{\sigma}(S) \subset \Gamma_{1} \underset{S}{\times} \cdots \underset{S}{\times} \Gamma_{\ell}, \\
& \begin{array}{l}
f \circ e+\sum_{|\sigma| \equiv 0} \operatorname{pr}_{1} \circ H \circ s^{\sigma}+\sum_{|\sigma| \equiv 1} \operatorname{pr}_{2} \circ H \circ s^{\sigma} \\
\quad=g \circ e+\sum_{|\sigma| \equiv 1} \operatorname{pr}_{1} \circ H \circ s^{\sigma}+\sum_{|\sigma| \equiv 0} \operatorname{pr}_{2} \circ H \circ s^{\sigma},
\end{array}
\end{aligned}
$$

where $s^{\sigma}=s_{1}^{(\sigma(1))} \times \cdots \times s_{\ell}^{(\sigma(\ell))}: S \rightarrow \Gamma_{1} \underset{S}{\times} \cdots \underset{S}{\times} \Gamma_{\ell}$ for $\sigma \in 2^{[1, \ell]}$.

Consider $h=(f, g): T \rightarrow C_{r}(V)_{d} \times C_{r}(V)_{d}$. The hypothesis implies $\operatorname{Im} h \subset E_{\ell}^{p}(V)_{d}$. Since $\operatorname{Im} h$ is constructible and $T$ is irreducible, there exists an irreducible open dense subset $T_{0}$ of $\operatorname{Im} h$. Since $E_{b}^{p}(V)_{d}^{\left(Q, \mu_{1}, \cdots, \mu_{\ell}, d^{\prime}, d^{\prime \prime}\right)}$ are constructible in $C_{r}(V)_{d} \times C_{r}(V)_{d}$ and since $k$ is uncountable, by virtue of (5.5.1), there exist $Q, \mu_{1}, \cdots, \mu_{\ell}, d^{\prime}$, and $d^{\prime \prime}$ such that $T_{0} \cap E_{\ell}^{p}(V)_{d}^{\left(Q, \mu_{1}, \cdots, \mu_{\ell}, d^{\prime}, d^{\prime \prime}\right)}$ contains a non-void open subset in $T_{0}$. Employing the above notations, consider

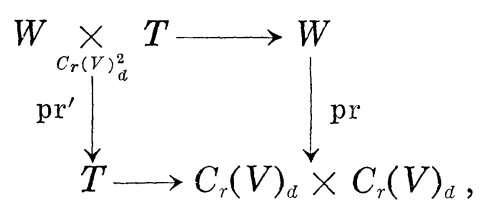

where $W$ is that in (5.3) for $Q, \mu_{1}, \cdots, \mu_{\xi}, d^{\prime}, d^{\prime \prime}$. Then $\mathrm{pr}^{\prime}$ is a dominant morphism; picking up some irreducible component $S^{\prime}$ of the fibre product dominating $T$ and replacing $S^{\prime}$ by some open subscheme of $S_{\text {red }}^{\prime}$, we may suppose that in the diagram

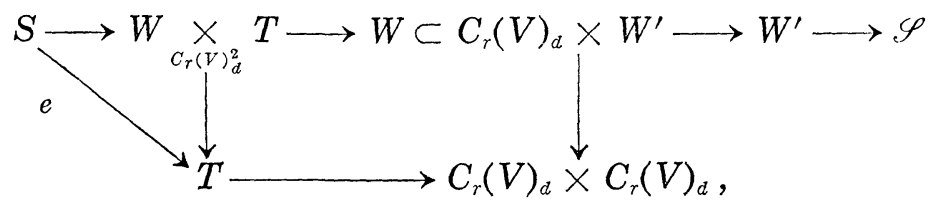

$S$ is smooth and $e: S \rightarrow T$ is dominant. By the base change $S \rightarrow \mathscr{S}$, we have families of curves (resp. jacobians) $\Gamma_{i}=\left(\mathscr{F}_{i}\right)_{S}$. Then the theorem follows from (5.4) and the commutativity of the diagram (5.6.3).

Theorem (5.7). Let $\kappa: T \rightarrow C H^{p}(V)$ be a regular map, where $T$ is a smooth quasiprojective variety. If $\kappa(T) \subset F^{\ell} C H^{p}(V)$, then, for $0 \leq \ell^{\prime}<\ell$, we have

$$
\kappa^{\sharp}\left(H^{r+\ell^{\prime}, r}(V)\right)=0,
$$

where $r+p=\operatorname{dim} V$. 
By the construction of $\kappa^{\#}$, it is sufficient to show that if $f, g: T \rightarrow$ $C_{r}(V)_{d}$ satisfy the hypothesis of (5.6), then $f^{\#} \omega=g^{\sharp} \omega$ for $\omega \in H^{r+\ell^{\prime}, r}(V)$. By virtue of (5.6), there exist a smooth quasi-projective variety $S$, a dominant morphism $e: S \rightarrow T$, families $\Gamma_{i} / S$ of jacobians, their sections $s_{\imath}^{(j)}: S \rightarrow \Gamma_{i}(1 \leq i \leq \ell ; j=0,1)$ and a rational map $H: \Gamma_{1} \underset{S}{\times} \cdots \underset{S}{\times} \Gamma_{\ell} \cdots$ $C_{r}(V)_{d^{\prime}} \times C_{r}(V)_{d^{\prime \prime}}$ satisfying the conditions (5.6.1) and (5.6.2). Note that $e^{*}: H^{0}\left(T, \Omega^{\ell^{\prime}}\right) \rightarrow H^{0}\left(S, \Omega^{\ell^{\prime}}\right)$ is injective since $e$ is dominant; it therefore suffices to verify $(f \circ e)^{\sharp} \omega=(g \circ e)^{\sharp} \omega$. The theorem will result from the next two lemmas, taking account of the property (2.4.3).

Lemma (5.7.1). Suppose $S$ and $T$ be smooth quasi-projective varieties and $\omega \in H^{r+\ell^{\prime}, r}(V)$. If $\varphi: T \cdots C_{r}(V)_{d}$ is a rational map, there exists an element $\eta \in H^{\ell^{\prime}, 0}(T)$ such that, for any morphism $f: S \rightarrow T$ with $f(S) \subset \operatorname{dom} \varphi$ (hence $\varphi \circ f$ is defined and a morphism), we have

$$
(\varphi \circ f)^{\sharp} \omega=f^{*} \eta \text {. }
$$

Let $T_{0}=\operatorname{dom} \varphi$ and $i: T_{0} \rightarrow T$ be the inclusion, $\varphi_{0}: T_{0} \rightarrow C_{r}(V)_{d}$ a representative of $\varphi, X$ the cycle on $T \times V$ corresponding to $\varphi$ and $X_{0}$ the cycle on $T_{0} \times V$ corresponding to $\varphi_{0}$. Then $\left(i \times \mathrm{id}_{V}\right)^{*} X$ is defined and equal to $X_{0}$. We set $\eta=X(\omega)$. Then, as shown in the proof of (2.4.2), we have $i^{*} \eta=X_{0}(\omega)$. The morphism $f$ as above is factorized as $f: S \stackrel{f_{0}}{\rightarrow} T_{0} \stackrel{i}{\rightarrow} T$. Then by definition, $\varphi \circ f=\varphi_{0} \circ f_{0}$, hence we have $(\varphi \circ f)^{\sharp} \omega=\left(\varphi_{0} \circ f_{0}\right)^{\sharp} \omega=f_{0}^{*}\left(\varphi_{0}{ }^{\sharp} \omega\right)$ $=f_{0}^{*}\left(X_{0}(\omega)\right)=f_{0}^{*}\left(i^{*}(\eta)\right)=f^{*} \eta$.

LEMMA (5.7.2). Let $S$ be a smooth quasi-projective variety, $\Gamma_{i} / S$ families of jacobian varieties $(1 \leq i \leq \ell)$, and $s_{i}^{(j)}: S \rightarrow \Gamma_{i}$ their sections $(1 \leq i \leq \ell$; $j=0,1)$. For any $\eta \in H^{\ell^{\prime}, 0}\left(\Gamma_{1} \underset{S}{\times} \cdots \times \Gamma_{S} \Gamma_{\ell}\right)$, if $\ell^{\prime}<\ell$, we have

$$
\sum_{\sigma}(-1)^{|\sigma|} s^{\sigma *}(\eta)=0 \quad \text { in } H^{\ell^{\prime}, 0}(S)
$$

Since we are dealing with a finite number of varieties and morphisms, and since $H^{\ell^{\prime}, 0}($ ?) commutes with the extension of the ground fields, we may assume $k=C$. Let $S^{h}, \Gamma_{i}^{h}$, etc. denote the complex analytic manifolds associated to $S, \Gamma_{i}$, etc. Since the diagram

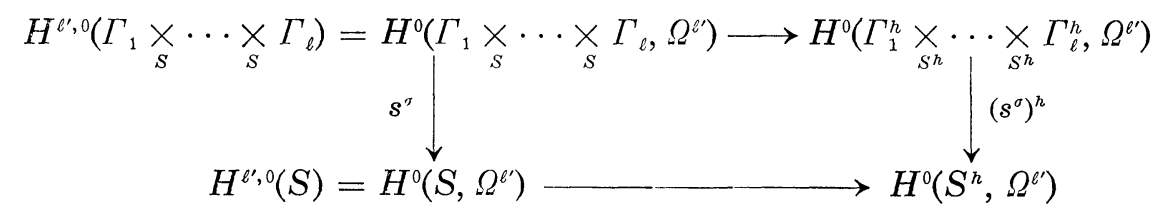


is commutative, where $\left(s^{\sigma}\right)^{h}=\left(s^{h}\right)^{\sigma}=\left(s_{1}^{(\sigma(1))}\right)^{h} \times \cdots \times\left(s_{\ell}^{(\sigma(\ell))}\right)^{h}$ and the horizontal maps are obvious ones, and since they are injective, it suffices to prove the lemma for the complex analytic version; we therefore omit the superscript $h$. The problem being local on $S$, we may suppose $S$ an open ball in $C^{m}, m=\operatorname{dim} S$. Since $\Gamma_{i} / S$ are families of jacobian varieties, $S \times C^{n_{i}}$ are the universal coverings of $\Gamma_{i}$, where $n_{i}=\operatorname{dim} \Gamma_{i}-m$. We get $S$-morphisms $S \times C^{n_{i}} \rightarrow \Gamma_{i}$ and

$$
p: S \times C^{n_{1}} \times \cdots \times C^{n_{\ell}}=\left(S \times C^{n_{1}}\right) \underset{S}{\times} \cdots \underset{S}{\times}\left(S \times C^{n_{\ell}}\right) \longrightarrow \Gamma_{1} \underset{S}{\times} \cdots \times \underset{S}{\times} \Gamma_{\ell} .
$$

Let $\tilde{s}_{i}^{(\jmath)}: S \longrightarrow S \times C^{n_{i}}$ be liftings of $s_{\imath}^{(j)}: S \rightarrow \Gamma_{i}$, and

$$
\tilde{s}^{\sigma}=\tilde{s}_{1}^{(\sigma(1))} \underset{S}{\times} \cdots \underset{S}{\times} \tilde{s}_{\ell}^{(\sigma(\ell))} .
$$

We have $\left(s^{\sigma}\right)^{*} \eta=\left(\tilde{s}^{\sigma}\right)^{*} \circ p^{*} \eta$. Let $z_{\imath}^{1}, \cdots, z_{i}^{n_{i}}$ be a coordinate system for $C^{n_{2}}$, and $x^{1}, \cdots, x^{m}$ for $S \subset C^{m}$. We set

$$
\begin{aligned}
d x^{I} & =d x^{i_{1}} \wedge \cdots \wedge d x^{i_{\lambda}} \\
d z_{i}^{J_{i}} & =d z^{j_{1}} \wedge \cdots \wedge d z^{j_{\nu}}
\end{aligned}
$$

for $I=\left\{i_{1}<\cdots<i_{\lambda}\right\}$ and $J_{i}=\left\{j_{1}<\cdots<j_{\nu}\right\}$. Then $p^{*} \eta$ is of the form

$$
p^{*} \eta=\sum f_{I, J_{1}, \ldots, J_{\ell}} d x^{I} \wedge d z_{1}^{J_{1}} \wedge \cdots \wedge d z_{\ell}^{J_{\ell}}
$$

where the summation is over $\left(I, J_{1}, \cdots, J_{\ell}\right)$ such that $I \subset\{1, \cdots, m\}, J_{i} \subset$ $\left\{1, \cdots, n_{i}\right\}$ with $|I|+\left|J_{1}\right|+\cdots+\left|J_{\ell}\right|=\ell^{\prime}$, and where $f_{I, J_{1}, \cdots, J_{\ell}} \in \Gamma\left(S \times C^{n_{1}} \times\right.$ $\left.\cdots \times C^{n_{\ell}}, \mathcal{O}\right)$. For $s \in S$ fixed, since $\left(\Gamma_{1} \times \cdots \times \Gamma_{\ell}\right)_{s}$ is compact, $f_{I, J_{1}, \cdots, J_{\ell}}(s, ?)$ are constants. So we may regard $f_{I, J_{1}, \ldots J_{\ell}} \in \Gamma(S, \mathcal{O})$. Denoting

$$
\bar{s}_{i}^{(j)}: S \stackrel{\tilde{s}_{i}^{(j)}}{\longrightarrow} S \times C^{n_{\iota}} \longrightarrow C^{n_{i}},
$$

and

$$
\zeta_{i}\left(\sigma, J_{i}\right)=\left(\bar{s}_{i}^{(\sigma(i))}\right) *\left(d z_{i}^{J i}\right),
$$

we have

$$
\left(\tilde{s}^{\sigma}\right)^{*} \circ p^{*} \eta=\sum f_{I, J_{1}, \ldots, J_{\ell}} d x^{I} \wedge \zeta_{1}\left(\sigma, J_{1}\right) \wedge \cdots \wedge \zeta_{\ell}\left(\sigma, J_{\ell}\right)
$$

Hence,

$$
\sum_{\sigma}(-1)^{|\sigma|}\left(s^{\sigma}\right)^{*} \eta=\sum f_{I, J_{1}, \ldots, J_{\ell}} d x^{I} \wedge\left[\sum_{\sigma}(-1)^{|\sigma|} \zeta_{1}\left(\sigma, J_{1}\right) \wedge \cdots \wedge \zeta_{\ell}\left(\sigma, J_{\ell}\right)\right],
$$

where by the first $\sum$ on the right side, we understand the summation over $\left(I, J_{1}, \cdots, J_{\ell}\right)$ as above. For a fixed $\left(I, J_{1}, \cdots, J_{\ell}\right)$, since $\left|J_{1}\right|+\cdots+\left|J_{\ell}\right|$ 
$=\ell^{\prime}-|I|<\ell$, there is a number $i$ such that $J_{i}=\phi$. Then the expression in the bracket is equal to zero because

$$
\begin{aligned}
\sum_{\sigma}(-1)^{|\sigma|} \zeta_{1}\left(\sigma, J_{1}\right) \wedge \cdots \wedge \zeta_{\ell}\left(\sigma, J_{\ell}\right) \\
=\sum_{\sigma(i)=0}(-1)^{|\sigma|} \zeta_{1}\left(\sigma, J_{1}\right) \wedge \cdots \wedge \widehat{\zeta_{i}\left(\sigma, J_{i}\right) \wedge \cdots \wedge \zeta_{\ell}\left(\sigma, J_{\ell}\right)} \\
\quad+\sum_{\sigma(i)=1}(-1)^{|\sigma|} \zeta_{1}\left(\sigma, J_{1}\right) \wedge \cdots \wedge \widehat{\zeta_{i}\left(\sigma, J_{i}\right) \wedge \cdots \wedge \zeta_{\ell}\left(\sigma, J_{\ell}\right)} \\
=0,
\end{aligned}
$$

where $\widehat{?}$ means the absence of ?. The lemma is thus proven.

Remark (5.8). We have proven the $\ell$-cubic equivalence versions of [9], Therems 1, 2 and 4. The results in [9, 10] deduced formally from them are, of course, valid in the $\ell$-cubic equivalence case. Here, we shall indicate two of such results (without proofs).

For any effective 0-cycle $X$ on $V$ of degree $d$ let

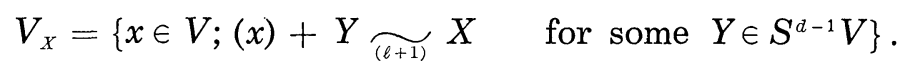

Then $V_{X}$ is a countable union of closed subsets of $V$. By $\operatorname{dim} V_{X}$, we understand the maximum of the dimensions of "irreducible components" of $V_{X}$ and let codim $V_{X}=\operatorname{dim} V-\operatorname{dim} V_{X}$. Then the set $\left\{X \in S^{a} V\right.$; codim $\left.V_{X}<a\right\}$ is a countable union of closed subsets of $S^{d} V$ for any integer $a$, so that there is a countable intersection $U$ of open subsets in $S^{d} V$ that the maximum $\delta_{d}$ of codim $V_{X}$ for $X \in S^{d} V$ is attained on $U$. Then $\delta_{d} \leq$ $\delta_{d+1}$ and we set

$$
d_{\ell}(V)=\lim _{d \rightarrow \infty} \delta_{d}=\delta_{d} \quad \text { for } d \gg 0 .
$$

Then $0 \leq d_{\ell}(V) \leq \operatorname{dim} V$ and $d_{\ell}(V) \neq 1$. Clearly, $d_{0}(V)=0$. By $(3.4,3)$ ), $d_{1}(V)=0$ if $V$ is a product of curves.

Corollary (5.8.1). If $H^{0}\left(V, \Omega^{q}\right) \neq 0, q \geq 2$, and $\ell \geq q$, then $d_{\ell}(V) \geq q$ (cf. [10], Theorem 3).

A canonical morphism $V \rightarrow$ Alb $V$ induces the natural map $F^{1} C H_{0}(V)$ Alb $V$, hence $g r^{1} C H_{0}(V) \rightarrow$ Alb $V$.

Corollary (5.8.2). If $d_{1}(V)=0$, then $g r^{1}(V) \rightarrow$ Alb $V$ is an isomorphism of groups (cf. [10], Theorem 4), and vice versa.

One can ask whether $d_{\ell}(V) \leq \ell$ or not for each $\ell$. If this is true for $\ell=1, g r^{1} C H_{0}(V)$ is always isomorphic to Alb $V$. For an abelian variety 
$V, d_{1}(V)=0$ and $g^{1} C_{0}(V) \simeq$ Alb $V$ (cf. [12]).

(5.9) To state the next theorem, we shall define ${ }^{\sharp} H^{p, q}(V)$.

Let $\mathfrak{U}^{p}(V)$ be the $k$-vector subspace of $H^{p, p}(V)$ generated by the fundamental classes of algebraic cycles on $V$ of codimension $p . \mathfrak{H}^{*}(V)=$ $\oplus_{p} \mathfrak{A}^{p}(V)$ is a subring of $H^{* *}(V)$ and stable by pull-backs or direct images. For a positive integer $\ell$, let $\left(H^{0,1}(V)\right)^{\cdot \ell}$ denote the image of

$$
\overbrace{H^{0,1}(V) \otimes \cdots \otimes H^{0,1}(V)}^{\ell \text { times }} \longrightarrow H^{0, \ell}(V)
$$

induced by the multiplication of $H^{* *}(V)$.

Definition (5.9.1). Let $0 \leq p \leq q$ be integers. ${ }^{\sharp} H^{p, q}(V)$ is by definition the $k$-vector subspace of $H^{p, q}(V)$ generated by the elements of the form $u(x)$, where $u \in \mathfrak{U}^{p+\operatorname{dim} T}(T \times V), x \in\left(H^{0,1}(T)\right)^{\cdot(q-p)}$ and $T$ is a smooth projective variety. Similarly, ${ }^{b} H^{p, q}(V)$ is the $k$-vector subspace generated by $u(x)$, where $u \in \mathfrak{A}^{p+\operatorname{dim} T}(T \times V)$ and $x \in H^{0, q-p}(T)$.

Clearly, ${ }^{\sharp} H^{p, q}(V) \subset{ }^{b} H^{p, q}(V)$ and ${ }^{\sharp} H^{p, p}(V)={ }^{b} H^{p, p}(V)=\mathfrak{A}^{p}(V)$.

Lemma (5.10). 1) For any morphism $f: U \rightarrow V, f^{*}\left({ }^{\sharp} H^{p, q}(V)\right) \subset{ }^{\sharp} H^{p, q}(U)$.

2) For any morphism $f: U \rightarrow V, f_{*}\left({ }^{\sharp} H^{p, q}(U)\right) \subset{ }^{\sharp} H^{p-d, q-d}(V)$, where $d=\operatorname{dim} U-\operatorname{dim} V$.

3) If $x \in{ }^{\sharp} H^{p, q}(U)$ and $y \in{ }^{\sharp} H^{p^{\prime}, q^{\prime}}(V)$, then $x \times y \in{ }^{\sharp} H^{p+p^{\prime}, q+q^{\prime}}(U \times V)$. The similar properties hold for ${ }^{b} H^{p, q}(V)$.

1) Indeed, for $v \in \mathfrak{A}^{*}(T \times V), y \in\left(H^{0,1}(T)\right)^{\cdot(q-p)}$, we have

$$
f^{*}(v(y))=\left(\left(\operatorname{id}_{T} \times f\right)^{*}(v)\right)(y) .
$$

2) For $u \in \mathfrak{Y}^{*}(T \times U), x \in\left(H^{0,1}(T)\right)^{\cdot(q-p)}$, we have

$$
f_{*}(u(x))=\left(\left(\operatorname{id}_{T} \times f\right)_{*}(u)\right)(x) .
$$

3) If $u \in \mathfrak{A}^{*}(T \times U), v \in \mathfrak{U}^{*}(S \times V), x \in\left(H^{0,1}(T)\right)^{\cdot(q-p)}$ and

$$
y \in\left(H^{0,1}(S)\right)^{\cdot\left(q^{\prime}-p^{\prime}\right)},
$$

then $u(x) \times v(y)=(u \times v)(x \times y), u \times v \in \mathfrak{2} *(T \times S \times U \times V)$ and

$$
x \times y \in\left(H^{0,1}(T \times S)\right)^{\cdot\left(q+q^{\prime}-p-p^{\prime}\right)} .
$$

Corollary (5.10.4). ${ }^{\sharp} H^{* *}(V)=\oplus_{0 \leq p \leq q}{ }^{\sharp} H^{p, q}(V)$ is a subring of $H^{* *}(V)$, and similarly for ${ }^{b} H^{* *}(V)$.

(5.11) We have clearly inclusions $\mathfrak{A} *(V) \subset{ }^{\sharp} H^{* *}(V) \subset{ }^{b} H^{* *}(V) \subset$ 
$H^{* *}(V)$. We shall show that in the definition of ${ }^{\sharp} H^{p, q}(V)$, we may restrict $T$ to varieties of the form

$$
T=C_{1} \times \cdots \times C_{q-p}, \quad C_{i}: \text { curves },
$$

or to abelian varieties. Suppose, in fact, given subspaces $\bar{H}^{p, q}(V)$ for all $p \leq q$, with the properties 1), 2), and 3) above and assume that $\bar{H}^{p, p}(V)$ $\supset \mathfrak{U}^{p}(V)$ and $\bar{H}^{0,1}(V)=H^{0,1}(V)$ for all $V$ and $p$. Then we have $H^{p, q}(V)$ $\supset{ }^{\sharp} H^{p, q}(V)$ for all $p \leq q$ by the properties 1$), 2$ ), and 3). Denote the $k$ vector subspace of $H^{p, q}(V)$ which, in the definition of ${ }^{\sharp} H^{p, q}(V)$, is obtained by restricting $T$ to abelian varieties (resp. products of curves as above) by $\bar{H}^{p, q}(V)$. Since $\bar{H}^{p, q}(V)$ has the properties 1 ), 2) and 3) above, and $\bar{H}^{p, p}(V)=\mathfrak{A}^{p}(V)$, we have only to check $\bar{H}^{0,1}(V)=H^{0,1}(V)$. In the case of $T$ being abelian varieties, if $i: V \rightarrow$ Alb $V=A$ is a canonical morphism, we have $i^{*}: H^{0,1}(A) \simeq H^{0,1}(V)$; but since $A$ is an abelian varieties, $H^{0,1}(A)$ $=\bar{H}^{0,1}(A)$, so that $H^{0,1}(V)=i^{*} H^{0,1}(A)=i^{*} \bar{H}^{0,1}(A) \subset \bar{H}^{0,1}(V) \subset H^{0,1}(V)$. In the case of $T$ being products of curves, it therefore suffices to show that $\bar{H}^{0,1}(V)=H^{0,1}(V)$ for an arbitrary abelian variety $V$. We can find a jacobian variety $J$ and a homomorphism $h: V \rightarrow J$ with finite kernel. Then since $h^{*} H^{0,1}(J)=H^{0,1}(V)$, we are reduced to the case where $V$ is the jacobian variety of a curve $C$ of genus $g$. We have a natural generically finite morphism $f: C^{g} \rightarrow V$. By projection formula,

$$
(\operatorname{deg} f) \mathrm{id}: H^{0,1}(V) \stackrel{f^{*}}{\longrightarrow} H^{0,1}\left(C^{g}\right) \stackrel{f_{*}}{\longrightarrow} H^{0,1}(V),
$$

hence $f_{*}$ is surjective. So if $\bar{H}^{0,1}\left(C^{g}\right)=H^{0,1}\left(C^{g}\right)$, we are done. The map

$$
K: H^{0,1}(C)^{\oplus g} \longrightarrow H^{0,1}\left(C^{g}\right)
$$

defined by

$$
K\left(x_{1}, \cdots, x_{g}\right)=\sum_{i=1}^{g} \operatorname{pr}_{i}^{*} x_{i}
$$

is an isomorphism, where $\mathrm{pr}_{i}: C^{g} \rightarrow C$ is the $i$-th projection. Since $H^{0,1}(C)=\bar{H}^{0,1}(C), H^{0,1}\left(C^{g}\right) \supset \bar{H}^{0,1}\left(C^{g}\right) \supset \operatorname{Im}\left(K: \bar{H}^{0,1}(C)^{\oplus g} \rightarrow H^{0,1}\left(C^{g}\right)\right)=\operatorname{Im} K$ $=H^{0,1}\left(C^{g}\right)$. By a similar method, we can show that, in the definition of ${ }^{b} H^{p, q}(V)$, we can restrict $T$ to varieties of dimension $q-p$.

Corollary (5.11.1). For any $V$ and $p,{ }^{\sharp} H^{p-1, p}(V)={ }^{b} H^{p-1, p}(V) \subset$ $H^{p-1, p}(V)$.

It is well-known that this subspace is equal to the tangent space of the algebraic part of the $p$-th intermediate jacobian of $V$ if $k=C$. 
Proposition (5.12). $\operatorname{dim}{ }^{\sharp} H^{0, q}(V)$ is a birational invariant of $V$ for each $q$.

If $V^{\prime} \rightarrow V$ is the blowing-up of $V$ with a non-singular center $W$, then $f^{*}:{ }^{\sharp} H^{0, q}(V) \rightarrow{ }^{\sharp} H^{0, q}\left(V^{\prime}\right)$ is an isomorphism. In fact we have an isomorphism

$$
H^{p, q}(V) \oplus \underset{0<i<r}{\oplus} H^{p-i, q-1}(W) \longrightarrow H^{p, q}\left(V^{\prime}\right),
$$

where $r$ is the codimension of $W$ in $V$, and this isomorpihsm and its inverse are composed of pull-backs, direct images and multiplications by fundamental classes of algebraic cycles. Hence the isomorphism remains valid when we replace $H$ by ${ }^{\sharp} H$. Since ${ }^{\sharp} H^{0-i, q-i}(W)=0$ for $i>0, f^{*}:{ }^{\sharp} H^{0, q}(V)$ $\simeq{ }^{\sharp} H^{0, q}\left(V^{\prime}\right)$. Now if $V$ and $V^{\prime}$ are birationally equivalent, we have a diagram

$$
V^{\prime} \longleftarrow V_{s} \longrightarrow \cdots \longrightarrow V_{1} \longrightarrow V_{0}=V
$$

where $V_{i+1}$ is a blowing up of $V_{i}$ with non-singular center, and $V_{s} \rightarrow V^{\prime}$ is a birational morphism. We have $\operatorname{dim}{ }^{\sharp} H^{0, q}\left(V^{\prime}\right) \leq \operatorname{dim}{ }^{\sharp} H^{0, q}\left(V_{s}\right)=\operatorname{dim}^{\sharp} H^{0, q}(V)$. Switching the roles of $V$ and $V^{\prime}$, we get the reversed inequality, hence $\operatorname{dim}^{\sharp} H^{0, q}(V)=\operatorname{dim}^{\sharp} H^{0, q}\left(V^{\prime}\right)$.

Remark (5.13). It is well-known that $\operatorname{dim}{ }^{b} H^{0, q}(V)=\operatorname{dim} H^{0, q}(V)$ is a birational invariant of $V$. We may ask whether $H^{0, q}(V)={ }^{\sharp} H^{0, q}(V)$ for any $V$ and $p$, which is equivalent to

$$
{ }^{b} H^{p, q}(V)={ }^{\sharp} H^{p, q}(V)
$$

for any $V$ and $p \leq q$.

Theorem (5.14). If ${ }^{\sharp} H^{p-\ell, p}(V) \neq 0$, then we have $\operatorname{gr}^{\ell} C H^{p}(V) \otimes \boldsymbol{Q} \neq 0$.

By hypothesis, we can find $\ell$ curves $C_{1}, \cdots, C_{\ell}, u \in \mathfrak{A}^{p+\ell}\left(C_{1} \times \cdots \times C_{\ell} \times V\right)$ and $x \in\left(H^{0,1}\left(C_{1} \times \cdots \times C_{\ell}\right)\right)^{\cdot \ell}=H^{0, \ell}\left(C_{1} \times \cdots \times C_{\ell}\right)$ such that $u(x) \neq 0$. We may further assume that $u$ is the fundamental class of a cycle $U$ on $C_{1} \times \cdots \times C_{\ell} \times V$. By abuse of notation, we shall denote the rational equivalence class of a cycle by the same symbol as the cycle itself. Fix points $a_{i}$ of $C_{i}(1 \leq i<\ell)$, and consider the regular maps

$$
\kappa^{\prime}: C_{1} \times \cdots \times C_{\ell} \longrightarrow \mathrm{CH}_{0}\left(C_{1} \times \cdots \times C_{\ell}\right)
$$

defined by

$$
\kappa^{\prime}\left(x_{1}, \cdots, x_{\ell}\right)=\left(\left(x_{1}\right)-\left(a_{1}\right)\right) \times \cdots \times\left(\left(x_{\ell}\right)-\left(a_{\ell}\right)\right) .
$$


and

$$
\kappa: C_{1} \times \cdots \times C_{\ell} \stackrel{\kappa^{\prime}}{\longrightarrow} C_{0}\left(C_{1} \times \cdots \times C_{\ell}\right) \stackrel{U(?)}{\longrightarrow} C^{p}(V) .
$$

$\operatorname{Im} \kappa^{\prime} \subset F^{\ell} C H_{0}\left(C_{1} \times \cdots \times C_{\ell}\right)$ implies $\operatorname{Im} \kappa \subset F^{\ell} C H^{p}(V)$. On the other hand, by (2.6.3), we have $\kappa^{\#}=\kappa^{\prime \prime}{ }^{t} u(?)$, where

$$
{ }^{t} u(?): H^{r+\ell, r}(V) \longrightarrow H^{\ell, 0}\left(C_{1} \times \cdots \times C_{\ell}\right) ;
$$

but ${ }^{t} u(?)$ is the transpose of

$$
u(?): H^{0, \ell}\left(C_{1} \times \cdots \times C_{\ell}\right) \longrightarrow H^{p-\ell, p}(V),
$$

hence we see ${ }^{t} u(?) \neq 0$. If we write $\kappa^{\prime}\left(x_{1}, \cdots, x_{\ell}\right)=\left(x_{1}\right) \times \cdots \times\left(x_{\ell}\right)+R$, then $R$ is a sum of elements of the form $\pm\left(y_{1}\right) \times \cdots \times\left(y_{\ell}\right)$, where at least one of $y_{i}^{\prime}$ 's is $a_{i}$ and the rests are $x_{i}^{\prime}$ 's. In other words,

$$
\kappa^{\prime}=\kappa_{0}+\sum \kappa_{j}
$$

where

$$
\kappa_{0}: C_{1} \times \cdots \times C_{\ell} \longrightarrow \mathrm{CH}_{0}\left(C_{1} \times \cdots \times C_{\ell}\right)
$$

is the regular map

$$
\kappa_{0}\left(x_{1}, \cdots, x_{\ell}\right)=\left(x_{1}\right) \times \cdots \times\left(x_{\ell}\right)
$$

and $\kappa_{j}$ are regular maps such that

$$
\kappa_{j}: C_{1} \times \cdots \times C_{\ell} \stackrel{f_{j}}{\longrightarrow} T_{j} \stackrel{\bar{\kappa}_{j}}{\longrightarrow} C H_{0}\left(C_{1} \times \cdots \times C_{\ell}\right)
$$

with $\bar{\kappa}_{j}$ regular, a morphism $f_{j}$, and $\operatorname{dim} T_{j}<\ell$. For $\eta \in H^{\ell, 0}\left(C_{1} \times \cdots C_{\ell}\right)$, we have $\kappa^{\prime \#} \eta=\kappa_{0}^{\sharp} \eta+\sum \kappa_{3}^{\sharp} \eta$. But $\kappa_{0}^{\sharp} \eta=\eta$, and $\kappa_{j}^{\#} \eta=0$, because

$$
\kappa_{j}^{\sharp}: H^{\ell, 0}\left(C_{1} \times \cdots \times C_{\ell}\right) \stackrel{\bar{\kappa}_{j}^{\sharp}}{\longrightarrow} H^{\ell, 0}\left(T_{j}\right) \stackrel{f_{j}^{*}}{\longrightarrow} H^{\ell, 0}\left(C_{1} \times \cdots \times C_{\ell}\right)
$$

and $\operatorname{dim} T_{j}<\ell$; thus $\kappa^{\sharp \#}=$ id on $H^{\ell, 0}\left(C_{1} \times \cdots \times C_{\ell}\right)$ so that $\kappa^{\sharp}={ }^{t} u(?) \neq 0$ on $H^{r+\ell, r}(V)$. For each integer $n>0,(n \kappa)^{\sharp}\left(H^{r+\ell, r}(V)\right) \neq 0$, which implies $\operatorname{Im}(n \kappa) \not \subset F^{\ell+1} C^{p}(V)$ in virtue of (5.7). Since

$$
\left\{c \in C_{1} \times \cdots \times C_{\ell} ; n \kappa(c) \in F^{\ell+1} C H^{p}(V)\right\}
$$

is a countable union of closed subsets of $C_{1} \times \cdots \times C_{\ell}$ distinct from $C_{1} \times \cdots \times C_{\ell}$ for each $n$ by (5.5.5),

$$
\bigcup_{n}\left\{c \in C_{1} \times \cdots \times C_{\ell} ; n \kappa(c) \in F^{\ell+1} C H^{p}(V)\right\} \neq C_{1} \times \cdots \times C_{\ell} .
$$

If $c \in C_{1} \times \cdots \times C_{\ell}$ does not belong to the first member, then for any 
$n>0, n \kappa(c) \notin F^{\ell+1} \mathrm{CH}^{p}(V)$, i.e., $\kappa(c) \in F^{\ell} \mathrm{CH}^{p}(V)$ gives a non-torsion ele.nent of $\mathrm{gr}^{\ell} C H^{p}(V)$, hence $\mathrm{gr}^{\ell} C H^{p}(V) \otimes \boldsymbol{Q} \neq 0$.

Remark (5.15). From ${ }^{b} H^{p-\ell, p}(V) \neq 0$, we deduce only

$$
\left(F^{1} C H^{p}(V) / F^{\epsilon+1} C H^{p}(V)\right) \otimes \boldsymbol{Q} \neq 0
$$

ExAMPLe (5.16). If $V$ is an abelian variety, we have $H^{0, p}(V)=$ $\wedge^{p} H^{0,1}(V)$, hence ${ }^{\sharp} H^{0, q}(V) \neq 0$ for $0 \leq q \leq \operatorname{dim} V$, which in turn implies

$$
{ }^{\sharp} H^{p, q}(V) \neq 0 \quad \text { for all } 0 \leq p \leq q \leq \operatorname{dim} V,
$$

by the Hard Lefschetz Theorem. Hence we find $\operatorname{gr}^{\ell} C H^{p}(V) \otimes \boldsymbol{Q} \neq 0$ if $0 \leq \ell \leq p \leq \operatorname{dim} V$. If $W$ is the associated Kummer variety, we have ${ }^{\sharp} H^{p, p}(W) \neq 0$ for $p+q \equiv 0$ (2), so that $\operatorname{gr}^{\ell} C H^{p}(W) \otimes \boldsymbol{Q} \neq 0$ if $\ell$ is even and $0 \leq \ell \leq q \leq \operatorname{dim} W$. Notice that ${ }^{\sharp} H^{p, q}(W)=0$ for $p+q \equiv 0$ (2); it would be interesting to know if $\operatorname{gr}^{\ell} \mathrm{CH}^{p}(W)=0$ for odd $\ell$.

\section{References}

[1] Bloch, S., Some elementary theorems about algebraic cycles on abelian varieties, Invent. Math., 37 (1975), 215-228.

[2] Deligne, P. and Mumford, D., The irreducibility of the space of curves of given genus. IHES, Publ. Math. $\mathrm{N}^{\circ} 36,75-109$.

[ 3 ] Griffiths, P. A., On the periods of certain rational integrals: I, II, Ann. of Math., 90 (1969), 460-495 and 496-541.

[4.] Grothendieck, A., La théorie des classes de Chern, Bull. Soc. Math. France, 86 (1958), 137-154.

[ 5 ] — and Dieudonne, J., Eléments de géometrie algébrique, IV (Troisième Partie). IHES, Publ. Math. $\mathrm{N}^{\circ} 28$.

[6] Hartshorne, R., Residue and duality. Lecture Notes in Math. 20, Springer 1966.

[ 7 ] Katz, N., Le Théorème de Griffiths. Lecture Notes in Math. 340, p. 341. Springer 1973.

[8] Mumford, D., Rational equivalence of 0-cycles on surfaces, J. Math. Kyoto Univ., 9 (1969), 195-204.

[ 9 ] Roitman, A. A., $\Gamma$-equivalence of zero-dimensional cycles, Mat. Sb., Tom 86 (128) (1971) $557-570=$ Math. USSR Sb., 15 (1971), 555-567.

[10] - Rational equivalence of zero-cycles, Mat. Sb. Tom 89 (131) (1972), 567-585 = Math. USSR Sb., 18 (1972), 571-588.

[11] Samuel, P., Methodes d'algébre abstraite en géometrie algébrique. Ergebniss, No. 4. Berlin-Göttingen-Heidelberg, Springer 1955.

[12] - - Relations d'équivalence en géométrie algébrique. Proc. Int. Congress Math. 1958, New York, Cambridge Univ. Press 1960.

[13] Weil, A., Sur les critères d'équivalence en géométrie algébrique, Math. Ann., 128 (1954), 95-127.

Department of Mathematics

Nagoya University

Nagoya, 464 Japan 